\title{
Right-veering diffeomorphisms of compact surfaces with boundary II
}

\author{
KO HONDA \\ WILLIAM H KAZEZ \\ GORDANA MATIĆ
}

\begin{abstract}
We continue our study of the monoid of right-veering diffeomorphisms on a compact oriented surface with nonempty boundary, introduced in [12]. We conduct a detailed study of the case when the surface is a punctured torus; in particular, we exhibit the difference between the monoid of right-veering diffeomorphisms and the monoid of products of positive Dehn twists, with the help of the Rademacher function. We then generalize to the braid group $B_{n}$ on $n$ strands by relating the signature and the Maslov index. Finally, we discuss the symplectic fillability in the pseudo-Anosov case by comparing with the work of Roberts $[15 ; 16]$.
\end{abstract}

57M50; 53C 15

\section{Introduction}

In [12], the authors introduced the study of right-veering diffeomorphisms on a compact oriented surface with nonempty boundary (sometimes called a "bordered surface"). This paper continues the investigations initiated in [12].

Let $\operatorname{Aut}(S, \partial S)$ be the isotopy classes of diffeomorphisms of a bordered surface $S$ which restrict to the identity on the boundary, $\operatorname{Veer}(S, \partial S)$ be the monoid of rightveering diffeomorphisms of $S$, and $\operatorname{Dehn}^{+}(S, \partial S)$ be the monoid of products of positive Dehn twists. (In particular, id is in both.) Recall that, by the work of Giroux [7], there is a 1-1 correspondence between isomorphism classes of open book decompositions modulo stabilization and isomorphism classes of contact structures on closed 3-manifolds. (Open books were introduced into contact geometry much earlier by Thurston and Winkelnkemper [17].) If $h \in \operatorname{Aut}(S, \partial S)$, let us write $(S, h)$ to denote, by slight abuse of notation, either the open book decomposition or the corresponding adapted contact structure. The main result of [12] is that a contact 3-manifold $(M, \xi)$ is tight if and only if all its adapted open book decompositions have right-veering monodromy. Here $M$ is closed and oriented, and $\xi$ is cooriented. On the other hand, Giroux [7] showed that $(M, \xi)$ is Stein fillable if and only if there 
is an adapted open book decomposition with monodromy $h \in \operatorname{Dehn}^{+}(S, \partial S)$. In order to understand the difference between tight and Stein fillable contact structures, as well as the symplectically fillable contact structures, which sit in between the two, we need to understand the difference between $\operatorname{Veer}(S, \partial S)$ and $\operatorname{Dehn}^{+}(S, \partial S)$.

One of the goals of this paper is to give an analysis of the difference between the monoids $\operatorname{Veer}(S, \partial S)$ and $\operatorname{Dehn}^{+}(S, \partial S)$ for the once-punctured torus $S$. The Rademacher function $\Phi$ and the rotation number rot, defined in Sections 2.2 and 2.3, taken together, are effective at distinguishing large swathes of $\operatorname{Veer}(S, \partial S)$ that are not in $\operatorname{Dehn}^{+}(S, \partial S)$. Our first theorem is the following:

Theorem 1.1 Let $S$ be a once-punctured torus and $h \in \operatorname{Aut}(S, \partial S)$. If $\operatorname{rot}(h) \geq 1 / 2$ and $-\Phi(h) \geq 10 \operatorname{rot}(h)$, then $h$ is in $\operatorname{Veer}(S, \partial S)$ but not in $\operatorname{Dehn}^{+}(S, \partial S)$.

Proof This follows from Lemma 2.4 and Theorem 2.3. (The lemma and the theorem are stated in terms of $\sigma$ in $B_{3}$, the braid group $B_{3}$ on 3 strands. See below for the discussion of $B_{3} \cong \operatorname{Aut}(S, \partial S)$.)

Theorem 2.3 is, to a large extent, a consequence of the fact that the linking number is positive on nontrivial elements of $\operatorname{Dehn}^{+}(S, \partial S)$. However, we also give evidence that the linking number is only a "first-order" invariant, in the sense that there are elements in $\operatorname{Veer}(S, \partial S)-\operatorname{Dehn}^{+}(S, \partial S)$ which cannot be measured by this technique, and require finer analysis. Examples of this are given in Section 2.5.

We will also generalize Theorem 2.3 to the case of the braid group $B_{n}$ on $n$ strands. If $S$ is a double branched cover of the disk, branched at $n$ points, then the hyperelliptic mapping class group HypAut $(S, \partial S)$ is the subgroup of $\operatorname{Aut}(S, \partial S)$, consisting of diffeomorphisms that commute with the hyperelliptic involution. Equivalently, it is the image of $B_{n}$ in $\operatorname{Aut}(S, \partial S)$. When $n=3, S$ is a punctured torus and half-twists about arcs connecting branch points lift to Dehn twists that generate $\operatorname{Aut}(S, \partial S)$. Hence $B_{3}$ can be identified with $\operatorname{HypAut}(S, \partial S)=\operatorname{Aut}(S, \partial S)$. On the other hand, for $n>3$, $\operatorname{Hyp} \operatorname{Aut}(S, \partial S)$ is a proper subgroup of $\operatorname{Aut}(S, \partial S)$.

Now, an element $\sigma \in B_{n}$ which is a product of conjugates of the standard positive half-twist generators is said to be quasipositive. The monoid of quasipositive braids corresponds to the monoid of products of positive Dehn twists, each of which is in HypAut $(S, \partial S)$. Observe that the monoid of quasipositive braids strictly contains the monoid of positive braids, ie, those which are positive products of the standard generators $\sigma_{1}, \ldots, \sigma_{n-1}$ of $B_{n}$. In Section 3 we prove Theorem 3.5, following the works of Gambaudo-Ghys [5; 4]. After lifting the action of $\operatorname{HypAut}(S, \partial S)$ on homology to $\widetilde{S p}(2 n, \mathbb{R})$, this theorem describes a relationship between the signature 
of the braid closure and the Maslov index of a corresponding "lift" to $\widetilde{S p}(2 n, \mathbb{R})$. Corollary 3.6 is then an incarnation of the fact that the linking number is positive on nontrivial quasipositive braids.

We then focus our attention to the question of which right-veering monodromy maps $h$ correspond to tight contact structures. In the pseudo-Anosov case we have the following result, which is proved in Section 4:

Theorem 1.2 Let $S$ be a bordered surface with connected boundary and $h$ be pseudoAnosov with fractional Dehn twist coefficient $c$. If $c \geq 1$, then $(S, h)$ is isotopic to a perturbation of a taut foliation. Hence $(S, h)$ is (weakly) symplectically fillable and universally tight if $c \geq 1$.

Hatcher [8] and Roberts [15; 16] constructed non-finite-depth taut foliations on certain Dehn fillings of punctured surface bundles. (Hatcher's work was for punctured torus bundles, which in turn was generalized by Roberts to all punctured surface bundles with one boundary puncture.) Theorem 1.2 follows from showing that the contact structure $(S, h)$ adapted to the open book is isotopic to perturbations of the Hatcher-Roberts taut foliations, using techniques developed in [11].

We are now left to analyze $(S, h)$ when $h$ is pseudo-Anosov and the fractional Dehn twist coefficient satisfies $0<c<1$. (Recall that if $c \leq 0$ then $(S, h)$ is overtwisted by Proposition 3.1 of [12].) For example, when $S$ is a punctured torus, we are concerned with $c=1 / 2$. In the paper [10], we prove, using Heegaard Floer homology, that $(S, h)$ is tight if $c=1 / 2$. This shows that if $S$ is a punctured torus and $h$ is pseudo-Anosov, then $(S, h)$ is tight if and only if $h \in \operatorname{Veer}(S, \partial S)$.

\section{$2 \operatorname{Veer}(S, \partial S)$ vs. $\operatorname{Dehn}^{+}(S, \partial S)$ on the punctured torus}

In this section we explain how to exhibit $h \in \operatorname{Veer}(S, \partial S)$ that are not products of positive Dehn twists, primarily via a combination of the Rademacher function and the rotation number.

\subsection{Preliminaries}

We discuss some preliminary notions, partly to fix terminology.

Let $S$ be the once-punctured torus and $T$ be the torus. There is a short exact sequence

$$
0 \rightarrow \mathbb{Z} \rightarrow \operatorname{Aut}(S, \partial S) \rightarrow \operatorname{Aut}(T) \rightarrow 1
$$


where the generator of $\mathbb{Z}$ is mapped to a positive Dehn twist $R_{\partial S}$ about $\partial S$. (In general, we use the notation $R_{\gamma}$ to denote a positive Dehn twist about a closed curve $\gamma$.) The group $\operatorname{Aut}(T)$ is isomorphic to $S L(2, \mathbb{Z})$, and is generated by

$$
A=\left(\begin{array}{cc}
0 & 1 \\
-1 & 0
\end{array}\right), B=\left(\begin{array}{cc}
1 & -1 \\
1 & 0
\end{array}\right) \text {. }
$$

Now, $\operatorname{Aut}(S, \partial S)$ can be identified with the (Artin) braid group $B_{3}$ on 3 strands. Denote the generators of $B_{3}$ by $\sigma_{1}$ and $\sigma_{2}$, corresponding to positive half-twists about strands 1, 2 and strands 2, 3. We then have the relation $\sigma_{1} \sigma_{2} \sigma_{1}=\sigma_{2} \sigma_{1} \sigma_{2}$. If we view the punctured torus as a 2 -fold branched cover of the disk with 3 branch points, then the positive half-twists on $B_{3}$ lift to positive Dehn twists on the punctured torus. More precisely, we choose the images $\bar{\sigma}_{i}$ of $\sigma_{i}$ in $S L(2, \mathbb{Z})$ to be

$$
\bar{\sigma}_{1}=\left(\begin{array}{cc}
1 & 0 \\
-1 & 1
\end{array}\right), \bar{\sigma}_{2}=\left(\begin{array}{ll}
1 & 1 \\
0 & 1
\end{array}\right) \text {. }
$$

Since $\bar{\sigma}_{1} \bar{\sigma}_{2} \bar{\sigma}_{1}=\bar{\sigma}_{2} \bar{\sigma}_{1} \bar{\sigma}_{2}$ in $S L(2, \mathbb{Z})$, the map $\sigma_{i} \mapsto \bar{\sigma}_{i}$ induces a homomorphism $B_{3} \rightarrow S L(2, \mathbb{Z})$. We have $A=\bar{\sigma}_{1} \bar{\sigma}_{2} \bar{\sigma}_{1}$ and $B=\bar{\sigma}_{1}^{-1} \bar{\sigma}_{2}^{-1}$. In the short exact sequence (2.1.1), $R_{\partial S} \mapsto\left(\sigma_{1} \sigma_{2} \sigma_{1}\right)^{4} \mapsto A^{4}$, which is the identity matrix in $\operatorname{SL}(2, \mathbb{Z})$.

Elements of $S L(2, \mathbb{Z})$ are grouped into three categories: reducible, periodic and Anosov. We will interpret the results from [12] to determine which elements $h$ of $\operatorname{Aut}(S, \partial S)$ are right-veering. By a slight abuse of terminology, we will often say " $h$ is Anosov" to mean " $\bar{h}$ is an Anosov diffeomorphism".

If $h$ is Anosov, then $\bar{h}$ has a pair of irrational eigenvalues $\lambda_{1}, \lambda_{2}$ that are both positive or both negative. In either case, there are two prongs of the stable lamination. If the $\lambda_{i}$ are positive, then the fractional Dehn twist coefficient $c$ is an integer $n$ and the prongs are fixed; if the $\lambda_{i}$ are negative, then $c$ is a half-integer $n+1 / 2$ and the prongs are switched. According to [12], an Anosov diffeomorphism $h$ is right-veering if and only if $c \geq 1 / 2$.

If $h$ is periodic, then $h$ is right-veering if and only if the fractional Dehn twist coefficient is $c \geq 0$. (Observe that $c=0$ corresponds to the identity diffeomorphism.) There is a short list of periodic elements in $S L(2, \mathbb{Z})$, up to conjugation:

$$
A_{1}=\left(\begin{array}{cc}
0 & 1 \\
-1 & 0
\end{array}\right), A_{2}=\left(\begin{array}{cc}
1 & 1 \\
-1 & 0
\end{array}\right), A_{3}=\left(\begin{array}{cc}
0 & 1 \\
-1 & -1
\end{array}\right)
$$

together with $-A_{1},-A_{2},-A_{3}$. The least right-veering lifts (they are right-veering, but leftmost amongst right-veering lifts) of $A_{i}$ are given by $a_{1}=\sigma_{1} \sigma_{2} \sigma_{1}, a_{2}=$ $\sigma_{1} \sigma_{2}$, and $a_{3}=\left(\sigma_{1} \sigma_{2}\right)^{2}$, respectively. These correspond to "rotations" by amounts $\pi / 2, \pi / 3,2 \pi / 3$ in the clockwise direction. (Hence $c$ must be in multiples of $1 / 4$ or 
1/6.) The least right-veering lifts of $-A_{i}$ are given by multiplying the above lifts $a_{i}$ by the central element $\left(\sigma_{1} \sigma_{2} \sigma_{1}\right)^{2}$, and the other right-veering lifts of $\pm A_{i}$ are $a_{i}\left(\sigma_{1} \sigma_{2} \sigma_{1}\right)^{2 k}, k \geq 0$. Notice that all the right-veering lifts are products of positive Dehn twists. Since any right-veering periodic $h$ is conjugate to one of the above lifts, $h$ must also be a product of positive Dehn twists. Hence, if $h$ is periodic, then $h \in \operatorname{Veer}(S, \partial S)$ if and only if $h \in \operatorname{Dehn}^{+}(S, \partial S)$. In other words, there is no difference between $\operatorname{Veer}(S, \partial S)$ and $\operatorname{Dehn}^{+}(S, \partial S)$ for periodic elements.

Finally, if $h$ is reducible, then $h$ can be written as $\left(\sigma_{1} \sigma_{2} \sigma_{1}\right)^{2 n} R_{\gamma}^{m}$, where $m$ and $n$ are integers, and $R_{\gamma}$ is a positive Dehn twist about some nonseparating curve $\gamma$. By Corollary 3.4 of [12] and the fact that $\operatorname{Veer}(S, \partial S) \subset \operatorname{Dehn}^{+}(S, \partial S)$ are monoids, we see that $h$ is right-veering if and only if either $n>0$, or $n=0$ and $m \geq 0$. Later we will show that if $n>0$ and $m \ll 0$, then $h$ is right-veering but not a product of positive Dehn twists.

\subsection{The Rademacher function}

Consider the action of $\operatorname{PSL}(2, \mathbb{Z})$ on the upper half-plane $\mathbb{H}^{2}$ and hence on the Farey tessellation of the unit disk $D^{2}$. Given

$$
\left(\begin{array}{ll}
a & b \\
c & d
\end{array}\right) \in P S L(2, \mathbb{Z})
$$

it acts on $\mathbb{H}^{2}$ by mapping

$$
z \mapsto \frac{c+d z}{a+b z} .
$$

In particular, if $z=p / q$ is a rational point on the $x$-axis, then

$$
\frac{p}{q} \mapsto \frac{c q+d p}{a q+b p}
$$

Under the correspondence

$$
\frac{p}{q} \leftrightarrow\left(\begin{array}{l}
q \\
p
\end{array}\right)
$$

the action of

$$
\left(\begin{array}{ll}
a & b \\
c & d
\end{array}\right)
$$

on $p / q$ is given by left multiplication on

$$
\left(\begin{array}{l}
q \\
p
\end{array}\right)
$$


Figure 1 shows the Poincare disk model for $\mathbb{H}^{2}$ with the points on $S_{\infty}^{1}$ labeled with the corresponding points on the $x$-axis of the upper half-plane model.

We now define the Rademacher function $\Phi: \operatorname{PSL}(2, \mathbb{Z}) \rightarrow \mathbb{Z}$. Much of what follows is taken from Barge-Ghys [1] and Gambaudo-Ghys [5; 4]. First observe that $P S L(2, \mathbb{Z})$ is isomorphic to the free product $\mathbb{Z} / 2 \mathbb{Z} * \mathbb{Z} / 3 \mathbb{Z}$, whose generators are $\pm A$ and $\pm B$. Hence any element $g \in P S L(2, \mathbb{Z})$ is uniquely written as $B^{r_{1}} A B^{r_{2}} \ldots B^{r_{k}}$, where $r_{1}, r_{k}=0,1$, or -1 and $r_{i}=-1$ or 1 , otherwise. We then define $\Phi(g)=\sum_{i=1}^{k} r_{i}$.

For a more geometric interpretation of $\Phi$, we will describe how $\Phi$ can be viewed as a function on the set of edges of the Farey tessellation. (See Figure 1.) We use the bijection between directed edges of the Farey tessellation and $P S L(2, \mathbb{Z})$, in which a directed edge $a \rightarrow b$ is identified with the element $g \in P S L(2, \mathbb{Z})$ which maps the slope 0 to $a$ and the slope $\infty$ to $b$. (In other words, $g$ is an orientation-preserving linear map which sends $(1,0)$ to a shortest integral vector with slope $a$ and $(0,1)$ to a shortest integral vector with slope $b$.) Notice that if $g$ corresponds to $a \rightarrow b$, then $g A$ corresponds to $b \rightarrow a$. Since right multiplication by $A$ does not change the value of $\Phi$, it follows that $\Phi$ induces a function on the set of (undirected) edges of the Farey tessellation. Undirected edges will be written as $a b$.

Again with $g$ corresponding to $a \rightarrow b$, choose $c$ so that $a, b$, and $c$ form the vertices of a triangle in clockwise order. Then $g B$ corresponds to $c \rightarrow a$ and $g B^{-1}$ corresponds to $b \rightarrow c$. Since the value of $\Phi$ on the identity map (or, equivalently, the edge $0 \rightarrow \infty$ ) is 0 , the value on the edge corresponding to any $g$ can be computed as follows. Let $p$ be a point on the edge $0 \rightarrow \infty$. Then $\Phi(g)$ equals the number of right turns minus the number of left turns for a geodesic from $p$ to $g(p)$. Here, a right turn refers to an arc from the edge $a b$ to $a c$ and a left turn refers to an arc from $a b$ to $b c$.

Remark In our definition of $\Phi$, we count the number of right turns minus the number of left turns. In $[1 ; 5]$, the authors count the number of left turns minus the number of right turns. The definitions of $\Phi: \operatorname{PSL}(2, \mathbb{Z}) \rightarrow \mathbb{Z}$ agree (at least on the set of hyperbolic elements), and the discrepancy is due to the difference in defining the action on $\mathbb{H}$. (If we defined $z=z_{1} / z_{2}$ instead of $z=z_{2} / z_{1}$, then we would be counting left turns minus right turns.)

One easily observes that $\Phi: \operatorname{PSL}(2, \mathbb{Z}) \rightarrow \mathbb{Z}$ is a quasi-morphism. A quasi-morphism is a map $\phi: G \rightarrow A$, where $G$ is a group and $A=\mathbb{Z}$ or $\mathbb{R}$, together with a constant $C$, such that $\left|\phi\left(g_{1} g_{2}\right)-\phi\left(g_{1}\right)-\phi\left(g_{2}\right)\right| \leq C$ for all $g_{1}, g_{2} \in G$. $\Phi$ is also not quite a homomorphism, as can be seen by taking $g_{1}$ which ends with $B$ and $g_{2}$ which begins with $B$. 


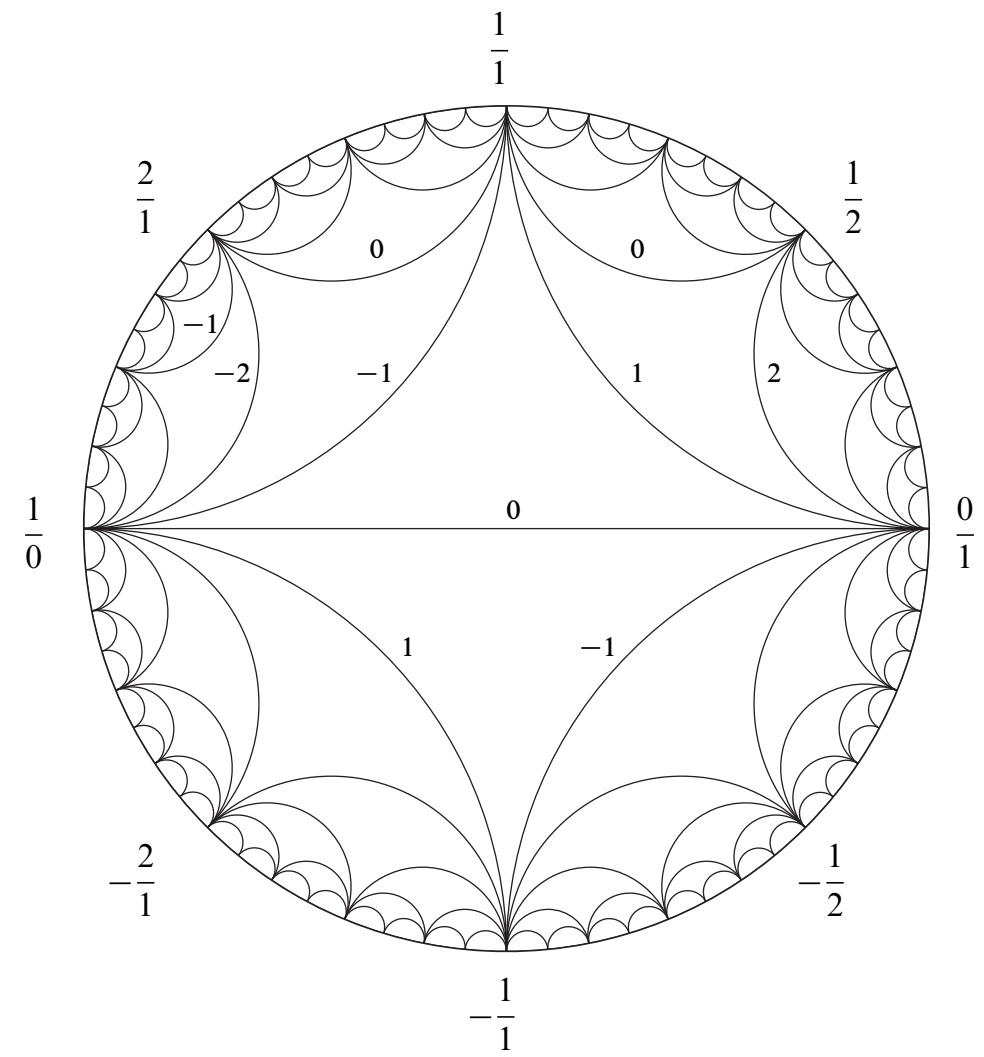

Figure 1: The Farey tessellation and values of the Rademacher function on the tessellation

\subsection{The linking number and rotation number}

Let $B_{n}$ be the braid group on $n$ strands. Then the linking number is a homomorphism lk: $B_{n} \rightarrow \mathbb{Z}$, defined as follows: if we write $\sigma \in B_{n}$ as $\sigma_{i_{1}}^{j_{1}} \ldots \sigma_{i_{k}}^{j_{k}}$, where $\sigma_{1}, \ldots, \sigma_{n-1}$ are the standard positive half-twists that generate $B_{n}$, then $\operatorname{lk}(\sigma)=j_{1}+\cdots+j_{k}$. The linking number $1 \mathrm{k}$ is a homomorphism because $B_{n}$ has relations only of the type $\sigma_{i} \sigma_{i+1} \sigma_{i}=\sigma_{i+1} \sigma_{i} \sigma_{i+1}$ and $\sigma_{i} \sigma_{j}=\sigma_{j} \sigma_{i}$, ie, those that leave the sums of the exponents constant. In fact, it is the unique homomorphism $B_{n} \rightarrow \mathbb{Z}$ (up to a constant multiple).

There is another invariant of $\operatorname{Aut}(S, \partial S) \simeq B_{3}$, which we will call the rotation number $\operatorname{rot}(h)$, which roughly measures the number of times $h$ rotates around $\partial S$. The normalization is such that $\operatorname{rot}\left(R_{\partial S}\right)=1$. Just as there is no homomorphism $\operatorname{Aut}(T) \rightarrow$ $\operatorname{Aut}(S, \partial S)$ which splits Equation (2.1.1), there are non-canonical choices involved in our definition of $\operatorname{rot}(h)$. 
Let $h \in \operatorname{Aut}(S, \partial S)$. We write $\sigma$ for the corresponding element in $B_{3}$, and $\bar{\sigma}$ or $a \rightarrow b$ for its image in $\operatorname{PSL}(2, \mathbb{Z})$. We consider four cases. (If $a \rightarrow b$ is $0 \rightarrow \infty$ (resp. $\infty \rightarrow 0$ ), then it is defined in Cases 1 and 4 (resp. Cases 2 and 3), and the two definitions agree.)

Case 1 If $0 \leq a<b \leq+\infty$, then we claim that $\sigma$ can be uniquely written as $\left(\sigma_{1} \sigma_{2} \sigma_{1}\right)^{2 n} w$, where $w$ is a word generated by $\sigma_{1}^{-1}$ and $\sigma_{2}$ and no inverses of these are allowed. First observe that $B A=\bar{\sigma}_{2}$ and $B^{-1} A=\bar{\sigma}_{1}^{-1}\left(\bar{\sigma}_{1} \bar{\sigma}_{2} \bar{\sigma}_{1}\right)^{2}=\bar{\sigma}_{1}^{-1}$. Since $0 \leq a<b \leq+\infty, a \rightarrow b$ can be written as $B^{ \pm 1} A B^{ \pm 1} A \ldots B^{ \pm 1} A$. (Recall the interpretation of $g A, g B$, and $g B^{-1}$ from Section 2.2, where $g \in P S L(2, \mathbb{Z})$ is viewed as a directed edge. The Farey tessellation and the dual graph indicate how to move the edge $0 \rightarrow \infty$ to $a \rightarrow b$.) This implies that $\bar{\sigma}$ can be written as a word $\bar{w}$ in $\bar{\sigma}_{1}^{-1}$ and $\bar{\sigma}_{2}$. The element $\sigma$ can therefore be written as $\left(\sigma_{1} \sigma_{2} \sigma_{1}\right)^{2 n} w$, by observing that the kernel of the projection $B_{3} \rightarrow P S L(2, \mathbb{Z})$ is generated by the central element $\left(\sigma_{1} \sigma_{2} \sigma_{1}\right)^{2}$. The uniqueness is a consequence of fact that the dual graph to the Farey tessellation is a tree (and hence there is a unique geodesic between any two vertices of the graph).

Case 2 If $-\infty \leq a<b \leq 0$, then $\sigma$ can uniquely be written as $\left(\sigma_{1} \sigma_{2} \sigma_{1}\right)^{2 n+1} w$, where $w$ is a word in $\sigma_{1}^{-1}$ and $\sigma_{2}$. Here, $a \rightarrow b$ can be written as $A\left(B^{ \pm 1} A B^{ \pm 1} A \ldots B^{ \pm 1} A\right)$.

Case 3 If $0 \leq b<a \leq+\infty$, then $\sigma$ can uniquely be written as $\left(\sigma_{1} \sigma_{2} \sigma_{1}\right)^{2 n+1} w$, where $w$ is a word in $\sigma_{1}$ and $\sigma_{2}^{-1}$. This is because $a \rightarrow b$ can be written as $B^{ \pm 1}\left(A B^{ \pm 1} A B^{ \pm 1} \ldots A B^{ \pm 1}\right)$ and $A B=\bar{\sigma}_{1}, A B^{-1}=\bar{\sigma}_{2}^{-1}$. Moreover, we have $B=\left(\bar{\sigma}_{1} \bar{\sigma}_{2} \bar{\sigma}_{1}\right)^{-1} \bar{\sigma}_{1}$ and $B^{-1}=\left(\bar{\sigma}_{1} \bar{\sigma}_{2} \bar{\sigma}_{1}\right) \bar{\sigma}_{2}^{-1}=\left(\bar{\sigma}_{2} \bar{\sigma}_{1} \bar{\sigma}_{2}\right) \bar{\sigma}_{2}^{-1}=\bar{\sigma}_{2} \bar{\sigma}_{1}$.

Case 4 If $-\infty \leq b<a \leq 0$, then $\sigma$ can uniquely be written as $\left(\sigma_{1} \sigma_{2} \sigma_{1}\right)^{2 n} w$, where $w$ is a word in $\sigma_{1}$ and $\sigma_{2}^{-1}$. Indeed, $a \rightarrow b$ can be written as $A B^{ \pm 1} A B^{ \pm 1} \ldots A B^{ \pm 1}$.

We now define the rotation number $\operatorname{rot}(\sigma)$ to be $k / 4$, where $\sigma=\left(\sigma_{1} \sigma_{2} \sigma_{1}\right)^{k} w$ as above.

Theorem 2.1 $1 \mathrm{k}(\sigma)=12 \operatorname{rot}(\sigma)+\mathrm{lk}(w)=12 \operatorname{rot}(\sigma)+\Phi(\bar{\sigma})$.

Proof Since $\mathrm{lk}$ is a homomorphism and $\sigma=\left(\sigma_{1} \sigma_{2} \sigma_{1}\right)^{4 \operatorname{rot}(\sigma)} w$ by the definition of the rotation number, the first equality follows. To see that $\operatorname{lk}(w)=\Phi(\bar{\sigma})$, first note that $\bar{\sigma}_{1}^{-1}=B^{-1} A$ and $\bar{\sigma}_{2}=B A$. If $w$ is a word in $\sigma_{1}^{-1}$ and $\sigma_{2}$, as is the case in Case 1, then the corresponding word in $A$ and $B^{ \pm 1}$ involves no cancellation of powers of $B$. It follows that $\operatorname{lk}(w)$, the exponent sum of the $\sigma_{1}$ 's and $\sigma_{2}$ 's, is the same as the exponent sum of the $B$ 's in the word corresponding to $w$; this, by definition, is $\Phi(\bar{w})$. 
Finally, since $\bar{\sigma}_{1} \bar{\sigma}_{2} \bar{\sigma}_{1}=(A B)(B A)(A B)=A$, powers of $\bar{\sigma}_{1} \bar{\sigma}_{2} \bar{\sigma}_{1}$ contribute nothing to the Rademacher function and therefore $\Phi(\bar{w})=\Phi(\bar{\sigma})$.

\section{$2.4 \operatorname{Veer}(S, \partial S)$ vs. $\operatorname{Dehn}^{+}(S, \partial S)$}

In this subsection we prove Theorem 2.3 and Lemma 2.4, which together comprise Theorem 1.1, and explore some consequences.

Observe that the linking number is useful in detecting braids which are not quasipositive:

Lemma 2.2 If one of the following holds, then $\sigma \in B_{n}$ is not quasipositive:

(1) $\operatorname{lk}(\sigma)<0$.

(2) $\operatorname{lk}(\sigma)=0$ but $\sigma \neq 1$.

(3) $\operatorname{lk}(\sigma)=1$ and $\sigma$ is not conjugate to a half-twist.

Proof This follows immediately from the fact that $\mathrm{k}$ is a homomorphism and consequently is constant on conjugacy classes.

Theorem 2.1 and (1) of Lemma 2.2 together imply that if $-\Phi(\bar{\sigma})>12 \operatorname{rot}(\sigma)$, then $\sigma$ is not quasipositive. We can actually do better:

Theorem 2.3 If $-\Phi(\bar{\sigma}) \geq 10 \operatorname{rot}(\sigma)$, then $\sigma$ is not quasipositive.

Proof We claim that if $\sigma=\sigma^{\prime \prime} \sigma^{\prime}$, where $\sigma^{\prime \prime}$ is a positive Dehn twist, then the triple $\left(\operatorname{lk}(\sigma)-\operatorname{lk}\left(\sigma^{\prime}\right), \operatorname{rot}(\sigma)-\operatorname{rot}\left(\sigma^{\prime}\right), \Phi(\bar{\sigma})-\Phi\left(\bar{\sigma}^{\prime}\right)\right)$ is one of $(1,0,1),(1,1 / 4,-2)$, or $(1,1 / 2,-5)$; moreover, if $\sigma^{\prime}=\mathrm{id}$, then only $(1,0,1)$ and $(1,1 / 4,-2)$ are possible. We then observe that $-\Phi(\bar{\sigma}) \leq 10 \operatorname{rot}(\sigma)$ if $\sigma$ is a product of positive Dehn twists. Since the first positive Dehn twist only contributes $(1,0,1)$ or $(1,1 / 4,-2)$, we find that the strict inequality $-\Phi(\bar{\sigma})=10 \operatorname{rot}(\sigma)$ is never attained.

The proof of the claim is a case-by-case analysis. Suppose $\bar{\sigma}$ is written as $a \rightarrow b$ and $\bar{\sigma}^{\prime}$ as $a^{\prime} \rightarrow b^{\prime}$. The slope of the Dehn twisting curve of $\sigma^{\prime \prime}$ is denoted by $c$. To visualize the action of this Dehn twist, consider the infinite collection of arcs of the Farey tessellation which end at $c$. The Dehn twist fixes the point $c$ and maps each arc clockwise to the next arc. Observe that the three cases below are sufficient, by reversing arrows or by taking negatives if necessary.

Remark The claim is intuitively reasonable if we consider the "amount of rotation about $\partial S$ " effected by each positive Dehn twist. The difficulty is that this quantity has a precise yet non-canonical meaning. We instead choose to keep track of $\Phi$, and the method of proof will be useful later in Section 2.5. 
Case 1 Suppose $a^{\prime} \rightarrow b^{\prime}$ is $0 \rightarrow \infty$.

If $c=\infty$, then $\bar{\sigma}$ is $-1 \rightarrow \infty$, and if $c=0$, then $\bar{\sigma}$ is $0 \rightarrow 1$. In both cases $\Phi$ changes by +1 , and hence rot by 0 , in view of Theorem 2.1 .

If $0<c<+\infty$, then let $c, d, e$ be the vertices of a triangle of the Farey tessellation in clockwise order, so that $a^{\prime} b^{\prime}$ and $d e$ are in the same connected component of $D^{2}$ cut open along $c d$ and $c e$. It could happen that $a^{\prime} b^{\prime}=d e$. If we apply a positive Dehn twist about $c$, then $c d e$ will be mapped to the adjacent triangle $c e f$. Let $P$ be a word in $L$ and $R$ which records the left and right turns taken on the geodesic from $0 \infty$ to $d e$. (For example, $L R L L$ means you first take a left turn and then a right turn, followed by two left turns.) If $P^{-1}$ is obtained from $P$ by reversing the word order and changing an $R$ to an $L$ and an $L$ to an $R$ (for example, if $P=L R L L$, then $P^{-1}=R R L R$ ), then the path from $0 \infty$ to $a b$ is given by $P L L P^{-1}$. See Figure 2 . $\Phi$ changes by -2 and hence rot by $1 / 4$. Similarly, if $-\infty<c<0$, then $\Phi$ changes by -2 and rot by $1 / 4$.

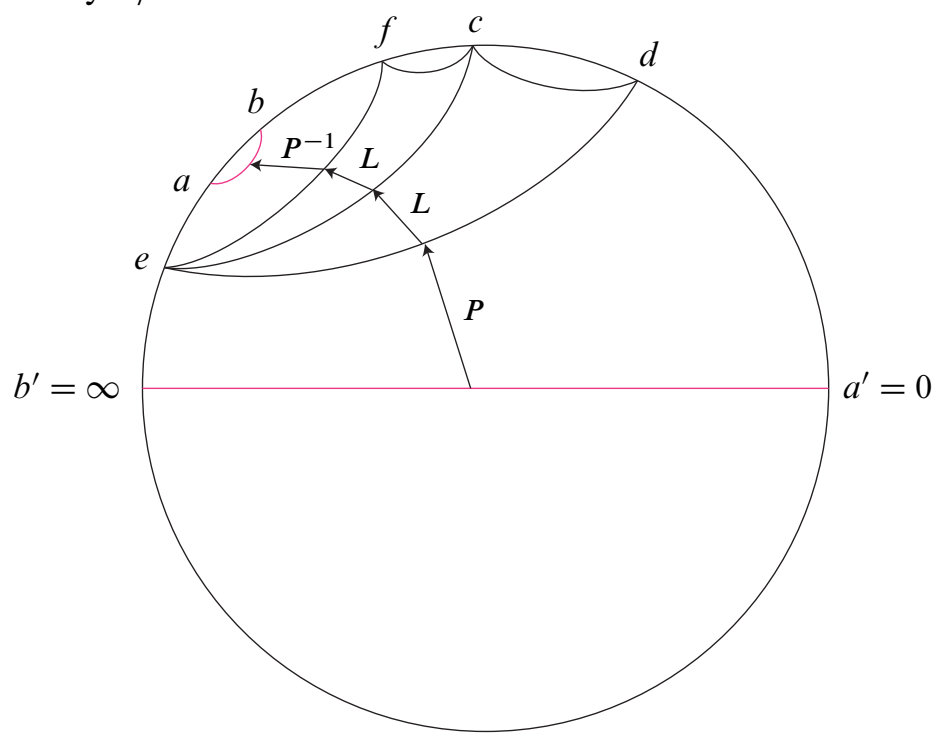

Figure 2: The path $P L L P^{-1}$ from $0 \infty$ to $a b$ in Case 1

Case 2 Suppose $0 \leq a^{\prime}<b^{\prime}<+\infty$.

If $c=a^{\prime}$, then $\Phi$ changes by +1 . If $c=b^{\prime}$, then $\Phi$ changes by +1 if $0 \leq a<a^{\prime}$, and by -2 if $b^{\prime}<a \leq+\infty$. If $a^{\prime}<c<b^{\prime}$, then $\Phi$ changes by -2 as in Case 1 .

If $0 \leq c<a^{\prime}$, then let $c d e$ be as above, ie, such that $a^{\prime} b^{\prime}$ and $d e$ are in the same connected component of $D^{2}$ cut open along $c d$ and $c e$, and let $c e f$ be the image 
of $c d e$ under the positive Dehn twist about $c$. Suppose first that $d \geq b^{\prime}$. If we draw a diagram like the one in Figure 2, we can see that the path from $0 \infty$ to $a^{\prime} b^{\prime}$ can be labeled by $P^{\prime} L Q$, where $P^{\prime}$ is the path from $0 \infty$ to $c d, L$ is the left turn at $d$, ie, from $c d$ to $d e$, and $Q$ is the path from $d e$ to $a^{\prime} b^{\prime}$. Then the path from $0 \infty$ to $a b$ is given by $P^{\prime} R L Q$. Here $R$ is a right turn around $c$ taking $d c$ to $c e$ and $L$ is a right turn around $e$ taking $c e$ to $e f$. We easily see that in this case $\Phi$ changes by +1 . Next suppose that $0 \leq d<c$. If $P_{1}$ is the path from $0 \infty$ to $a^{\prime} b^{\prime}$, and $P_{2}$ is the path from $a^{\prime} b^{\prime}$ to $d e$, then we can write the paths as $P_{1}=P_{1}^{\prime} L Q$ and $P_{2}=Q^{-1} L P_{2}^{\prime}$. Then the path from $0 \infty$ to $a b$ is given by $\widehat{P_{1} P_{2}} L L P_{2}^{-1}$, where indicates a contraction. More precisely, since $P_{1}=P_{1}^{\prime} L Q$ and $P_{2}=Q^{-1} L P_{2}^{\prime}$, where the two $L$ 's are around two vertices of the same triangle, we get $\widehat{P_{1} P_{2}}=P_{1}^{\prime} R P_{2}^{\prime}$, where $R$ corresponds to the right turn at the third vertex. In the end the path from $0 \infty$ to $a b$ is $P_{1}^{\prime} R P_{2}^{\prime} L L P_{2}^{-1}=P_{1}^{\prime} R P_{2}^{\prime} L L\left(P_{2}^{\prime}\right)^{-1} R Q$. Recalling that $P_{1}=P_{1}^{\prime} L Q$, we see that $\Phi$ changes by +1 as well.

Suppose $-\infty<c<0$. If the path from $0 \infty$ to $a^{\prime} b^{\prime}$ is $P_{1}$, then the path from $0 \infty$ to $a b$ is of the form $P_{2} L L P_{2}^{-1} P_{1}$. Therefore, $\Phi$ changes by -2 .

Next suppose $b^{\prime}<c<+\infty$. If $a^{\prime} \geq e>f \geq 0$, then the path from $0 \infty$ to $a^{\prime} b^{\prime}$ is given by $P_{1} L R P_{2}$, where $L$ and $R$ are turns through $c e f$ and $c d e$. Then the path from $0 \infty$ to $a b$ is given by $P_{1} R P_{2}$, and $\Phi$ changes by +1 . If $a^{\prime} \geq e \geq 0$ and $f>c$, then $a, b$ satisfies one of the following: (i) $e \geq a>b \geq 0$, (ii) $-\infty \leq a, b \leq 0$, (iii) $a, b \geq f$, or (iv) $e \geq a \geq 0$ and $b>f$. In any case, we can write $P_{1} R R P_{2}$ for the path from $0 \infty$ to $a^{\prime} b^{\prime}$, where $P_{1}$ is the path from $0 \infty$ to $e f$, the $R$ 's rotate about $e$ and $P_{2}$ is the path from $d e$ to $a^{\prime} b^{\prime}$. In case (i), the word $P_{1} R R P_{2}$ for the path from $0 \infty$ to $a^{\prime} b^{\prime}$ is transformed to $\widehat{P_{1} P_{2}}$. More precisely, we can write $P_{1}=P_{1}^{\prime} L Q$ and $P_{2}=Q^{-1} L P_{2}^{\prime}$, and we get $P_{1}^{\prime} R P_{2}^{\prime}$. So $\Phi$ changes by +1 . In (ii), the word $P_{1} R R P_{2}$ is transformed to $P_{2}^{\prime}$, where $P_{2}=P_{1}^{-1} P_{2}^{\prime}$. $\Phi$ changes by -2 . In (iii), $P_{1} R R P_{2}$ is transformed to $\widehat{P_{1} P_{2}}$, where $P_{1}=P_{1}^{\prime} R Q$ and $P_{2}=Q^{-1} R P_{2}^{\prime}$ and $\widehat{P_{1} P_{2}}=P_{1}^{\prime} L P_{2}^{\prime}$. This time $\Phi$ changes by -5 . In (iv), $P_{1} P_{2} R R P_{2}^{-1}$ is transformed to $P_{1}$, and $\Phi$ changes by -2 . If $e>f>c, P_{1}$ is the path from $0 \infty$ to $a^{\prime} b^{\prime}$ and $P_{2}$ is the path from $a^{\prime} b^{\prime}$ to $d e$, then the path from $0 \infty$ to $a b$ is $\widehat{P_{1} P_{2}} L L P_{2}^{-1}$, and $\Phi$ changes by -5 .

Finally take $c=\infty$. If $c d e=\infty 10$, then $R P$ maps to $L P$ and $\Phi$ changes by -2 . Otherwise, $P_{1} L R P_{2}$ maps to $P_{1} R P_{2}$ and $\Phi$ changes by +1 .

Case 3 Suppose $a^{\prime} \rightarrow b^{\prime}$ is $a^{\prime} \rightarrow \infty$, where $a^{\prime}$ is a nonnegative integer.

If $c=\infty$ or $c=a^{\prime}$, then $\Phi$ changes by +1 . If $a^{\prime}<c<+\infty$, then $\Phi$ changes by -2 as in Case 1. If $0 \leq c<a^{\prime}$, then $\Phi$ changes by +1 as in Case 2. Finally, if $-\infty<c<0$, then $\Phi$ changes by -2 as in Case 2. Notice that in this case $\Phi$ cannot change by -5 . 
Theorem 2.3 is effective when used in conjunction with the following lemma:

Lemma 2.4 If $\operatorname{rot}(\sigma) \geq 1 / 2$, then $\sigma$ is right-veering.

Before proceeding with the proof, we briefly discuss the action of $h \in \operatorname{Aut}(S, \partial S)$ on the universal cover $\widetilde{S}$ of $S$, as described in [12]. In this paragraph we assume that the Euler characteristic $\chi(S)$ is negative, ie, $S$ is not a disk or an annulus. Endow $S$ with a hyperbolic metric for which $\partial S$ is geodesic. The universal cover $\pi: \widetilde{S} \rightarrow S$ can then be viewed as a subset of the Poincaré disk $D^{2}=\mathbb{H}^{2} \cup S_{\infty}^{1}$. Now let $L$ be a component of $\pi^{-1}(\partial S)$. If $h \in \operatorname{Aut}(S, \partial S)$, choose a lift $\tilde{h}$ of $h$ that is the identity on $L$. The closure $\widetilde{S}$ in $D^{2}$ is geodesically convex. One portion of $\partial \widetilde{S}$ is $L$ and the complement of the closure of $L$ in $\partial \widetilde{S}$ will be denoted $L_{\infty}$. Note that $L_{\infty}$ is homeomorphic to $\mathbb{R}$. Orient $L_{\infty}$ using the boundary orientation of $\widetilde{S}$ and then linearly order $L_{\infty}$ so that moving in an orientation-preserving sense increases the order. The lift $\tilde{h}$ induces a homeomorphism $h_{\infty}: L_{\infty} \rightarrow L_{\infty}$. By Theorem 2.2 of [12], $h$ is right-veering if and only if $z \geq h_{\infty}(z)$ for all $z \in L_{\infty}$.

Proof of Lemma 2.4 This can be proved on a case-by-case basis, as in the definition of the rotation number in Section 2.3. We will treat Case 1, ie, $0 \leq a<b \leq+\infty$, and leave the other cases to the reader. Since $\operatorname{rot}(\sigma) \geq 1 / 2$, we have $\sigma=\left(\sigma_{1} \sigma_{2} \sigma_{1}\right)^{2 n} w$, where $n \geq 1$. It suffices to verify the lemma for $n=1$. In the rest of the proof we write $\sigma$ if we mean an element in $B_{3}$, and write $h$ to denote the corresponding element in $\operatorname{Aut}(S, \partial S)$.

Using the notation from the paragraph preceding the proof, pick a basepoint $x \in \partial S$ and a lift $\tilde{x} \in L$. We can endow $L_{\infty}$ with a nondecreasing continuous function $\theta: L_{\infty} \rightarrow \mathbb{R}$ so that any properly embedded, oriented $\operatorname{arc} \alpha:[0,1] \rightarrow S$ with $\alpha(0)=x$ and slope $s$ satisfies $\theta(\widetilde{\alpha}(1)) \equiv \theta_{s}(\bmod 2 \pi)$, where $\widetilde{\alpha}$ is a lift of $\alpha$ to $\widetilde{S}$ whose initial point is $\tilde{x}$ and $\theta_{s}$ is the standard angle that a line of slope $s$ makes with a line of slope 0 . (Here $\theta$ would be an angular coordinate on $K_{\infty}$, obtained from $L_{\infty}$ by quotienting each connected component of $\pi^{-1}(\partial S)$ besides $L$ to a point.)

Let $\alpha$ and $\beta$ be properly embedded, oriented arcs based at $x$ with slopes $a$ and $b$, such that $0 \leq \theta(\widetilde{\alpha}(1))<\theta(\widetilde{\beta}(1)) \leq \pi / 2$. The element $h_{\infty}$ maps the interval $[0, \pi / 2]$ to $[\theta(\widetilde{\alpha}(1))-\pi, \theta(\widetilde{\beta}(1))-\pi]$ and maps $[-\pi / 2,0]$ to $[\theta(\widetilde{\beta}(1))-2 \pi, \theta(\widetilde{\alpha}(1))-\pi]$. By applying the same argument to other intervals, we see that $\sigma$ is right-veering. The other cases are similar.

Remark Observe that, in order to show that $\sigma$ is right-veering, it is not sufficient to verify that two properly embedded arcs of $S$ corresponding to an integer basis of $\mathbb{Z}^{2}$ get mapped to the right. 
In the rest of the subsection we give some consequences of the above discussion.

Corollary 2.5 For the punctured torus $S$, there are infinitely many pseudo-Anosov diffeomorphisms $h \in \operatorname{Veer}(S, \partial S)$ with arbitrarily large fractional Dehn twist coefficients $c$, which are not in $\operatorname{Dehn}^{+}(S, \partial S)$.

Proof As before, we switch freely between $\sigma \in B_{3}$ and its corresponding $h \in$ $\operatorname{Aut}(S, \partial S)$. Choose $\sigma=\left(\sigma_{1} \sigma_{2} \sigma_{1}\right)^{2 n} w$ with $n \geq 1$ as in Case 1 , but with $0<a<$ $b<+\infty$. Then, the action of $\bar{\sigma} \in P S L(2, \mathbb{Z})$ on the circle at infinity $S_{\infty}^{1}$ of the Farey tessellation has two fixed points. Therefore $\bar{\sigma}$ is Anosov and $h \in \operatorname{Aut}(S, \partial S)$ is pseudo-Anosov. (Alternatively, one can compute the trace of $\bar{\sigma}$, and show that it is $>2$ or $<-2$, since the entries are all positive or all negative.) Since $n \geq 1$, all such $h$ are right-veering by Lemma 2.4. On the other hand, if $w$ is chosen so that $\#\left(\sigma_{1}^{-1}\right.$ terms $)-\#\left(\sigma_{2}\right.$ terms $)$ is sufficiently large, then $h \notin \operatorname{Dehn}^{+}(S, \partial S)$.

To rephrase Corollary 2.5 in terms of the braid group $B_{n}$, we recall Thurston's left orderings of $B_{n}$. Let $S$ be the double branched cover of the unit disk, branched along $n$ points. Thurston defined left orderings of $\operatorname{Aut}(S, \partial S)$ (and hence the left orderings on $B_{n}$ ) via the double branched cover $S$ : Fix $z \in L_{\infty}$. Given $h, g \in \operatorname{Aut}(S, \partial S)$, define $h \geq_{z} g$ if $h(z) \geq g(z)$. Such an ordering is called a left ordering because it preserved by left multiplication. (Of course, $\geq_{z}$ may not be a total order, but that is not an important issue here.) The following is a rephrasing of Corollary 2.5.

Corollary 2.6 There exist infinitely many pseudo-Anosov braids $\sigma \in B_{3}$ for which id $\geq \sigma$ using any of the left orderings of $B_{3}$ defined by Thurston, but which are not quasipositive.

Example 2.7 Let $\sigma=\left(\sigma_{1} \sigma_{2} \sigma_{1}\right)^{2} \sigma_{1}^{-m}$. Then

$$
\bar{\sigma}=\left(\begin{array}{cc}
-1 & 0 \\
-m & -1
\end{array}\right) \text {. }
$$

This is the right-veering lift of $\bar{\sigma} \in S L(2, \mathbb{Z})$ with the least amount of rotation to the right. By Theorem 2.3, if $m \geq 5$, then $\sigma$ is not quasipositive. On the other hand, we claim that, for $m \leq 4, \sigma$ can be written as a product of positive Dehn twists. It suffices to prove the claim for $\left(\sigma_{1} \sigma_{2} \sigma_{1}\right)^{2} \sigma_{1}^{-4}$. Indeed using the fact that

$$
\left(\sigma_{1} \sigma_{2} \sigma_{1}\right) \sigma_{1}=\sigma_{2}\left(\sigma_{1} \sigma_{2} \sigma_{1}\right),\left(\sigma_{1} \sigma_{2} \sigma_{1}\right) \sigma_{2}=\sigma_{1}\left(\sigma_{1} \sigma_{2} \sigma_{1}\right)
$$

we write:

$$
\begin{aligned}
\left(\sigma_{1} \sigma_{2} \sigma_{1}\right)^{2} \sigma_{1}^{-4} & =\left(\sigma_{1} \sigma_{2} \sigma_{1}\right)\left(\sigma_{1} \sigma_{2} \sigma_{1}^{-1}\right) \sigma_{1}^{-2} \\
& =\left(\sigma_{2} \sigma_{1} \sigma_{2}^{-1}\right)\left(\sigma_{1} \sigma_{2} \sigma_{1}\right) \sigma_{1}^{-2}=\left(\sigma_{2} \sigma_{1} \sigma_{2}^{-1}\right)\left(\sigma_{1} \sigma_{2} \sigma_{1}^{-1}\right) .
\end{aligned}
$$


More generally, we can show that $\left(\sigma_{1} \sigma_{2} \sigma_{1}\right)^{2 n} \sigma_{1}^{-m}$ is quasipositive if $m \leq 4 n$ but not quasipositive if $m \geq 5 n$. In general, we do not know what happens for $m$ strictly between $4 n$ and $5 n .^{1}$

Corollary 2.8 There does not exist a finite set of generators for $\operatorname{Veer}(S, \partial S)$ over $\operatorname{Aut}(S, \partial S)$, that is, there is no finite collection $C$ of elements of $\operatorname{Veer}(S, \partial S)$ such that every element of $\operatorname{Veer}(S, \partial S)$ can be expressed as a product of positive powers of elements of $C \cup \operatorname{Dehn}^{+}(S, \partial S)$.

Proof Consider $\sigma(m)=\left(\sigma_{1} \sigma_{2} \sigma_{1}\right)^{2} \sigma_{1}^{-m}$ with $m \geq 5$. By Example 2.7, $\sigma(m) \in$ $\operatorname{Veer}(S, \partial S)-\operatorname{Dehn}^{+}(S, \partial S)$. The homeomorphism $h_{\infty}(m): L_{\infty} \rightarrow L_{\infty}$ corresponding to $\sigma(m)$ sends $[0, \pi / 2]$ to $\left[\tan ^{-1}(m)-\pi, \pi / 2-\pi\right]$. Notice that every angle is decreased by at most $\pi$, and the only angles that are decreased by $\pi$ are $\pi / 2+k \pi$.

We claim that if $h_{1}, h_{2} \in \operatorname{Veer}(S, \partial S)$ and $\sigma(m)=h_{1} h_{2}$, then one of the $h_{i}$ is $\sigma\left(m^{\prime}\right)$ with $m^{\prime} \geq m$ and the other is $\sigma_{1}^{m^{\prime}-m}$; the corollary then follows immediately from the claim. Effectively we are showing that the $\sigma(m)$ are the least right-veering among right-veering diffeomorphisms which are not in $\operatorname{Dehn}^{+}(S, \partial S)$.

Arguing by contradiction, let $\sigma(m)=h_{1} h_{2}$ be such a factorization. Since $\sigma(m) \notin$ $\operatorname{Dehn}^{+}(S, \partial S)$, it is not possible that both $h_{1}, h_{2} \in \operatorname{Dehn}^{+}(S, \partial S)$. First we claim that $h_{i}$ cannot be freely homotopic to a pseudo-Anosov homeomorphism. Indeed, for a pseudo-Anosov $h_{i}$ to be right-veering, it must have fractional Dehn twist coefficient $c \geq 1 / 2$ by Proposition 3.1 of [12]. Then there is a properly embedded $\operatorname{arc} \alpha$ on $S$ so that $\theta(\widetilde{\alpha}(1))-\theta(\widetilde{h(m)}(1))>\pi$. (Take $\alpha$ so that its slope is close to, but slightly larger than, the stable slope.) Since homeomorphisms which are freely homotopic to periodic homeomorphisms in $\operatorname{Veer}(S, \partial S)$ are necessarily in $\operatorname{Dehn}^{+}(S, \partial S)$, it follows that one of the factors $h_{i}$ must be reducible and not in $\operatorname{Dehn}^{+}(S, \partial S)$. This means that $h_{i}$ can be expressed as $\left(\sigma_{1} \sigma_{2} \sigma_{1}\right)^{2 n_{1}} R_{\gamma}^{n_{2}}$. Since $h_{i}$ is right-veering, $n_{1} \geq 0$, but $n_{1}=0$ would imply $n_{2}>0$ and then $h_{i} \in \operatorname{Dehn}^{+}(S, \partial S)$. Also $n_{1}$ cannot be greater than or equal to 2 , since the angle of rotation would be too large, and $h_{i}$ could not be a factor of $\sigma(m)$. This leaves the possibility $h_{i}=\left(\sigma_{1} \sigma_{2} \sigma_{1}\right)^{2} R_{\gamma}^{n_{2}}$. In this case the only angles that are decreased by $\pi$ when acted on by $\left(\sigma_{1} \sigma_{2} \sigma_{1}\right)^{2} R_{\gamma}^{n_{2}}$ are the angles corresponding to $\pm \gamma$. It follows that $\gamma$ has slope $\infty$; thus $h_{i}=\sigma\left(-n_{2}\right)$. Letting $m^{\prime}=-n_{2}$ and using the fact that $h_{i}$ decreases angles by no more than $\sigma(m)$ implies $m^{\prime} \geq m$.

\footnotetext{
${ }^{1}$ The referee has informed us that the following holds: For any $m$, the least $\Psi(m)$ for which $\left(\sigma_{1} \sigma_{2} \sigma_{1}\right)^{\Psi(m)} \sigma_{1}^{-m}$ is quasipositive is $\Psi(m)=2 k+1$ for $m=5 k, 5 k+1,5 k+2$, and $\Psi(m)=2 k+2$ for $m=5 k+3,5 k+4$. In each case, $\left(\sigma_{1} \sigma_{2} \sigma_{1}\right)^{\Psi(m)-1} \sigma_{1}^{-m}$ is not quasipositive by Theorem 2.3, so Theorem 2.3 gives a tight bound for parabolic elements.
} 


\subsection{An example}

In this subsection, we will give a computation of an element $h \in \operatorname{Veer}(S, \partial S)-$ $\operatorname{Dehn}^{+}(S, \partial S)$ which does not satisfy the condition of Theorem 2.3. It is likely that the types of computations done in the example are amenable to computer calculation, ie, the algorithm can probably be done in finite time for the torus.

Example 2.9 $\sigma=\left(\sigma_{1} \sigma_{2} \sigma_{1}\right)^{2} \sigma_{1}^{-4} \sigma_{2} \sigma_{1}^{-1} \sigma_{2} \sigma_{1}^{-1}$ is in $\operatorname{Veer}(S, \partial S)-\operatorname{Dehn}^{+}(S, \partial S)$. However, $\Phi=-4$, rot $=1 / 2$ and $\mathrm{lk}=2$, and the conditions of Theorem 2.3 are not satisfied. Our strategy is to exploit the fact that $1 \mathrm{k}=2$, so that $\sigma$ must be expressed as a product of two positive Dehn twists if $\sigma \in \operatorname{Dehn}^{+}(S, \partial S)$. There are two possibilities: (1) the first Dehn twist contributes $(1,0)$ to $(\Phi$, rot $)$ and the second contributes $(-5,1 / 2)$, or $(2)$ the first contributes $(-2,1 / 4)$ and the second $(-2,1 / 4)$.

(1) Referring to the proof of Theorem 2.3, Case 1, the first Dehn twist sends $0 \rightarrow \infty$ to $0 \rightarrow 1$ or $-1 \rightarrow \infty$. By looking at the $\sigma$ we are considering, we see that the image is in the upper half disk of the Farey tessellation; let us denote the corresponding word by $W$. By inspecting again the proof of Theorem 2.3 we see that if the second Dehn twist contributes $(-5,1 / 2)$, then it leaves $0 \rightarrow 1$ or $-1 \rightarrow \infty$ in the same half disk (upper or lower) of the Farey tessellation, so $-1 \rightarrow \infty$ is not possible. In the only possible combination of twists we easily see that if $\bar{\sigma}$ is written as $a^{\prime} \rightarrow b^{\prime}$, then the path $W$ from $0 \infty$ to $a^{\prime} b^{\prime}$ can be written as $L P L L P^{-1} L$, where $P$ is some word in $L$ and $R$. This is a contradiction.

(2) The first Dehn twist maps $0 \rightarrow \infty$ to $a \rightarrow b$, where (a) $0<b<a<+\infty$ or (b) $-\infty<a<b<0$. Suppose the second Dehn twist maps $a \rightarrow b$ to $a^{\prime} \rightarrow b^{\prime}$. In case (a), there are three relevant subcases: (i) the slope $s_{2}$ of the second Dehn twist satisfies $b<s_{2}<a$; (ii) $s_{2}>a$ and $a^{\prime} \leq b, b^{\prime} \geq a$; (iii) $s_{2}=a$ and $b^{\prime}>a$. This again follows from the analysis of the proof of Theorem 2.3. In subcase (i), $W$ can be written as $P_{1} L L P_{1}^{-1} P_{2} L L P_{2}^{-1}$. In subcase (ii), after analyzing all the possible diagrams, we see that the only relative position of the two adjacent triangles in the Farey tessellation with vertex $s_{2}$ that results in a contribution of $(-2,1 / 4)$ is the one presented in Figure 3 . Moreover, the edge $a^{\prime} \rightarrow b^{\prime}$ can be any edge which intersects the geodesic from $0 \infty$ to the lower triangle with vertex $s_{2}$ given in Figure 3. One particular possibility for $a^{\prime} \rightarrow b^{\prime}$ is given in Figure 3; this gives $W=P_{2} L L P_{2}^{-1} P_{1}^{-1} L L P_{1}$. The other possibilities for $a^{\prime} \rightarrow b^{\prime}$ are edges of the two triangles with vertex $s_{1}$ and edges between $0 \infty$ and the lower triangle with vertex $s_{1}$; they give equations $W=P L L P^{-1} L L$, $W=L P L L P^{-1} L, W=L L P L L P^{-1}$, and $W P_{1}=P_{1} L L P_{2} L L P_{2}^{-1}$. In subcase (iii), $W$ can be written as $L P L L P^{-1} L$. In case (b), there are also two subcases: $s_{2}>0$ or $b<s_{2} \leq 0$. In the former subcase, $W$ can be written as $P_{2} L L P_{2}^{-1} P_{1} L L P_{1}^{-1}$. In the latter, we can write $P_{1} W=P_{2} L L P_{2}^{-1} L L P_{1}$. 


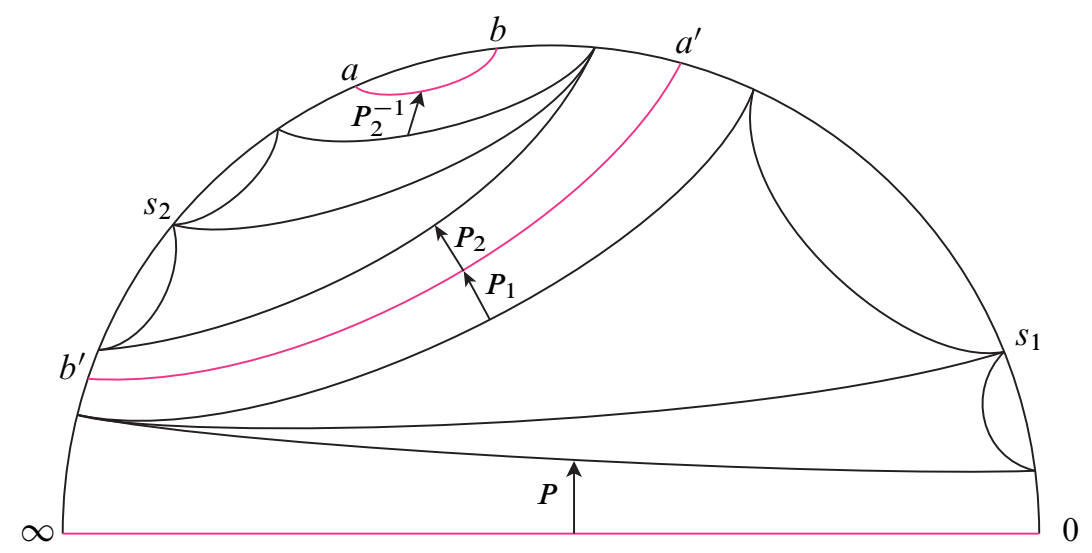

Figure 3: Case (ii)(a) with $s_{2}>a$. Here $P=P_{2} L L P_{2}^{-1} P_{1}^{-1}$, and $W=P L L P_{1}$.

In summary, if $W$ is the path from $0 \infty$ to $a^{\prime} b^{\prime}$, we need to show that each of the following equations has no solution:

$$
\begin{aligned}
L P L L P^{-1} L & =W \\
P_{1} L L P_{1}^{-1} P_{2} L L P_{2}^{-1} & =W \\
P_{2} L L P_{2}^{-1} L L P_{1} & =P_{1} W \\
P_{1} L L P_{2} L L P_{2}^{-1} & =W P_{1}
\end{aligned}
$$

So far we have only used the facts that $\Phi=-4$, rot $=1 / 2$ and $\mathrm{lk}=2$. We now show that our specific choice $W=L L L L R L R L=L^{4} R L R L$ has no solution to any of the above equations. The first two equations are immediate. To see that the third equation has no solution note that we can write it in the form $X P=P W$ where $P=P_{1}$. Since $P$ must have the same last letter as $W$, we can write $P=Q Y$ and $W=Z Y$ (for example, we can take $Y=R L R L$ ). Then $X Q=Q Y Z$. Notice that this is an equation of the same form as $X P=P W$, but with $Q$ repeated instead of $P$, and that $Q$ is shorter than $P$ and $Y Z$ is a cyclic permutation of $W$. Notice also that $Q$ is not the empty word since no cyclic permutation of $W$ is equal to $X$, regardless of the choice of $P_{2}$. This means the argument can be repeated, ie, $Q$ must have the same last letter as $Z$ and can be written as $Q=Q_{1} Y_{1}$ with $Y Z=Z_{1} Y_{1}$, etc. This procedure inductively shortens $Q_{i}$. Since we can never reduce to the empty word, this gives us a contradiction. The fourth equation can be treated in the same way as the third.

In general, this line of argument can be done for many words. Long sequences with $R L R L R L \ldots$ are effective, since the equations above all contain $L L$. 


\subsection{Questions}

We close this section with some questions.

Suppose $S$ is the punctured torus. We were able to identify large swathes of the complement $\operatorname{Veer}(S, \partial S)-\operatorname{Dehn}^{+}(S, \partial S)$. However, we are far from determining all of $\operatorname{Veer}(S, \partial S)-\operatorname{Dehn}^{+}(S, \partial S)$.

Question 2.10 Determine a complete set of invariants that will distinguish elements of $\operatorname{Veer}(S, \partial S)$ that are not in $\operatorname{Dehn}^{+}(S, \partial S)$.

Our initial motivation for undertaking the study of the difference between $\operatorname{Veer}(S, \partial S)$ and $\operatorname{Dehn}^{+}(S, \partial S)$ was to understand the difference between tight contact structures and Stein fillable contact structures. The following question is still open.

Question 2.11 If $h \in \operatorname{Veer}(S, \partial S)-\operatorname{Dehn}^{+}(S, \partial S)$ for $S$, then is $(S, h)$ not Stein fillable? Is it true for a general bordered surface $S$ ? Is it true if $S$ is a punctured torus?

There is some evidence that the answer is yes, which the authors learned from Giroux. Recall that $(S, h)$ is Stein fillable if and only if there is some $\left(S^{\prime}, h^{\prime}\right) \in \operatorname{Dehn}^{+}(S, \partial S)$ so that the open books $(S, h)$ and $\left(S^{\prime}, h^{\prime}\right)$ become the same after performing a sequence of stabilizations to each (no destabilizations allowed). The work of Orevkov [13] shows that, in the braid group (or, equivalently, in the hyperelliptic mapping class group), $\sigma \in B_{n}$ is quasipositive if and only if its stabilization in $B_{n+1}$ is quasipositive. It is not clear to the authors how to adapt Orevkov's argument to the more general situation.

By Orevkov, all the $h \in \operatorname{Veer}(S, \partial S)-\operatorname{Dehn}^{+}(S, \partial S)$ constructed above for the punctured torus $S$ are still not quasipositive when stabilized and viewed in the braid group/hyperelliptic mapping class group. Moreover, after a certain number of stabilizations, the linking number of the braid is no longer negative!

\section{Generalizations to the braid group}

We now discuss generalizations of the results from the previous section to the braid group $B_{n}$.

One method is to start with $\sigma \in B_{3}$ which is right-veering but not in $\operatorname{Dehn}^{+}(S, \partial S)$ because $1 \mathrm{k}<0$, and then embed $\rho: B_{3} \hookrightarrow B_{n}$ (somewhat) canonically by adding extra strands. Since $\operatorname{Veer}(S, \partial S)$ is a monoid, one can take products of such $\rho(\sigma)$, their conjugates in $B_{n}$, and quasipositive elements $\sigma^{\prime} \in B_{n}$. Provided the linking number is still negative, the product is right-veering but not quasipositive. 
Another method (presumably slightly more general) is to rephrase the $1 \mathrm{k}<0$ condition in terms of the signature of the braid closure and the Maslov index. This uses, in an essential way, the work of Gambaudo-Ghys [5; 4]. After some preparatory remarks in Sections 3.1 and 3.3, we prove Theorem 3.5 in Section 3.4.

\subsection{Bounded cohomology}

In this subsection we interpret Theorem 2.3 in terms of bounded cohomology.

Since much of the material is probably unfamiliar to specialists in contact and symplectic geometry, we include a brief summary of bounded cohomology and the (bounded) Euler class. An excellent source is Ghys [6].

Let $G$ be a group and $A=\mathbb{Z}$ or $\mathbb{R}$. Then the (ordinary) cohomology group $H^{k}(G ; A)$ is the cohomology of the chain complex $\left(C^{k}(G ; A), \delta\right)$, where $C^{k}(G ; A)$ is the set of maps $c: G^{k+1} \rightarrow A$ which are homogeneous, ie, $c\left(g g_{0}, g g_{1}, \ldots, g g_{k}\right)=$ $c\left(g_{0}, \ldots, g_{k}\right)$, and $\delta: C^{k-1}(G ; A) \rightarrow C^{k}(G ; A)$ is the cochain map:

$$
\delta c\left(g_{0}, g_{1}, \ldots, g_{k}\right)=\sum_{i=0}^{k}(-1)^{i} c\left(g_{0}, \ldots, \hat{g}_{i}, \ldots, g_{k}\right) .
$$

The bounded cohomology group $H_{b}^{k}(G ; A)$ is the cohomology of the chain complex $C_{b}^{k}(G ; A) \subset C^{k}(G ; A)$ of maps $c: G^{k+1} \rightarrow A$ for which

$$
\left|\sup _{\left(g_{0}, \ldots, g_{k}\right) \in G^{k+1}} c\left(g_{0}, \ldots, g_{k}\right)\right|<\infty .
$$

There is a natural map $H_{b}^{k}(G ; A) \rightarrow H^{k}(G ; A)$ which is not necessarily injective or surjective.

The homogeneous cochain $c: G^{k+1} \rightarrow A$ corresponding to the inhomogeneous cochain $\bar{c}: G^{k} \rightarrow A$ is given by $c\left(g_{0}, \ldots, g_{k}\right)=\bar{c}\left(g_{0}^{-1} g_{1}, g_{1}^{-1} g_{2}, \ldots, g_{k-1}^{-1} g_{k}\right)$. In the other direction, we can find the inhomogeneous cochain $\bar{c}$ whose homogenization is $c$ by setting $\bar{c}\left(h_{1}, \ldots, h_{k}\right)=c\left(e, h_{1}, h_{1} h_{2}, h_{1} h_{2} h_{3}, \ldots, h_{1} h_{2} \ldots h_{k}\right)$. With this dehomogenization, the coboundary of $C^{1}(G ; A)$ is defined on inhomogeneous maps by

$$
\delta \bar{c}\left(h_{1}, h_{2}\right)=\bar{c}\left(h_{1}\right)+\bar{c}\left(h_{2}\right)-\bar{c}\left(h_{1} h_{2}\right) .
$$

What we are interested in is $H^{2}(G ; A)$, which classifies isomorphism classes of central extensions of $G$ by $A$ :

$$
0 \rightarrow A \rightarrow \widetilde{G} \rightarrow G \rightarrow 1
$$


The class in $H^{2}(G ; A)$ corresponding to the central extension given by Equation (3.1.1) is called the Euler class of the central extension.

Now define a quasi-morphism to be a map $\phi: G \rightarrow A$, together with a constant $C$, such that $\left|\phi\left(g_{1} g_{2}\right)-\phi\left(g_{1}\right)-\phi\left(g_{2}\right)\right| \leq C$ for all $g_{1}, g_{2} \in G$. Denote by $Q M(G ; A)$ the $A$-module of quasi-morphisms from $G$ to $A$. A trivial quasi-morphism is a quasi-morphism $\phi$ which is a bounded distance from a genuine homomorphism $\psi$, ie, $\phi-\psi$ is bounded on $G$. (Hence, two quasi-morphisms are deemed equivalent if their difference is within bounded distance of a genuine homomorphism.) The following fact can be verified directly from the definitions.

Fact The kernel of $H_{b}^{2}(G ; A) \rightarrow H^{2}(G ; A)$ is the quotient of $Q M(G ; A)$ by the trivial quasi-morphisms.

\subsection{Interpretation of Theorem 2.1 from the viewpoint of bounded coho- mology.}

Let Homeo+ $\left(S^{1}\right)$ be the group of orientation-preserving homeomorphisms of $S^{1}$ and $\widehat{\mathrm{Homeo}}_{+}\left(S^{1}\right)$ be the universal cover of $\mathrm{Homeo}_{+}\left(S^{1}\right)$. If we identify $S^{1}=\mathbb{R} / \mathbb{Z}$, then an element $\tilde{\gamma}$ of $\widetilde{\mathrm{HomeO}}_{+}\left(S^{1}\right)$ is a periodic orientation-preserving homeomorphism of $\mathbb{R}$ with period 1 . Define the translation number $\Psi: \widetilde{\text { Homeo }}_{+}\left(S^{1}\right) \rightarrow \mathbb{R}$, where $\Psi(\tilde{\gamma})=2 \tilde{\gamma}(0)$ if $\tilde{\gamma}(0) \in \mathbb{Z}$ and $2\lfloor\tilde{\gamma}(0)\rfloor+1$ if $\tilde{\gamma}(0) \notin \mathbb{Z}$. Here $\lfloor\cdot\rfloor$ is the greatest integer function. The translation number, roughly speaking, keeps track of twice the number of times a point is sent around $S^{1}$. The translation number $\Psi$ is a quasi-morphism of $\widehat{\mathrm{Homeo}}_{+}\left(S^{1}\right)$, whose coboundary

$$
\delta \Psi\left(g_{1}, g_{2}\right)=\Psi\left(g_{1}\right)+\Psi\left(g_{2}\right)-\Psi\left(g_{1} g_{2}\right)
$$

descends to Homeo $\left(S^{1}\right)$ and represents an element in the second bounded cohomology group $H_{b}^{2}\left(\mathrm{Homeo}_{+}\left(S^{1}\right) ; \mathbb{Z}\right)$.

Via the standard action of $\operatorname{PSL}(2, \mathbb{R})$ on $\mathbb{R} \mathbb{P}^{1} \simeq S^{1}$, we may view $\operatorname{PSL}(2, \mathbb{R})$ as a subgroup of $\mathrm{Homeo}_{+}\left(S^{1}\right)$ and $\widetilde{P S L}(2, \mathbb{R})$ as a subgroup of $\widetilde{\mathrm{Homeo}}_{+}\left(S^{1}\right)$. Here $\widetilde{P S L}(2, \mathbb{R})$ is viewed as the group of equivalence classes of paths in $\operatorname{PSL}(2, \mathbb{R})$ starting at the identity. Also let $\widetilde{P S L}(2, \mathbb{Z}) \subset \widetilde{P S L}(2, \mathbb{R})$ be the equivalence classes of paths in $\operatorname{PSL}(2, \mathbb{R})$ starting at id and ending at an element in $\operatorname{PSL}(2, \mathbb{Z})$. We now restrict $\Psi$ to $\overparen{P S L}(2, \mathbb{Z})$, which is isomorphic to $\operatorname{Aut}(S, \partial S)$, where $S$ is the once-punctured torus. Recall that any $\sigma \in B_{3} \cong \operatorname{Aut}(S, \partial S)$ can uniquely be written as $\left(\sigma_{1} \sigma_{2} \sigma_{1}\right)^{k} w$, where $w$ is a product of $\sigma_{1}, \sigma_{2}^{-1}$ or $\sigma_{1}^{-1}, \sigma_{2}$. Since $\Psi$ and -4 rot agree on all powers of $\left(\sigma_{1} \sigma_{2} \sigma_{1}\right)^{4}$, they differ by a bounded amount on $\overparen{P S L}(2, \mathbb{Z})$. 
Hence their coboundaries $\delta \Psi$ and $\delta(-4$ rot $)$ represent the same element in the bounded cohomology group $H_{b}^{2}(P S L(2, \mathbb{Z}) ; \mathbb{Z})$.

Next, given the Rademacher function $\Phi: P S L(2, \mathbb{Z}) \rightarrow \mathbb{Z}$, consider its coboundary $\delta \Phi$. Although $\delta \Phi$ is zero in the ordinary group cohomology $H^{2}(P S L(2, \mathbb{Z}) ; \mathbb{Z})=$ $\mathbb{Z} / 6 \mathbb{Z}$, it is nevertheless a nontrivial element in the bounded cohomology group $H_{b}^{2}(P S L(2, \mathbb{Z}) ; \mathbb{Z})$ : First observe that $\Phi$ is not a bounded 1 -cochain. Moreover, since $\operatorname{PSL}(2, \mathbb{Z}) \cong \mathbb{Z} / 2 \mathbb{Z} * \mathbb{Z} / 3 \mathbb{Z}$, there is no nonzero homomorphism $\operatorname{PSL}(2, \mathbb{Z}) \rightarrow \mathbb{Z}$. (Observe that there is no nonzero homomorphism from $\mathbb{Z} / m \mathbb{Z}$ to $\mathbb{Z}$, when $m$ is a positive integer.) Hence $\Phi$ is not a bounded distance from any homeomorphism and therefore represents a nontrivial element in $H_{b}^{2}(P S L(2, \mathbb{Z}) ; \mathbb{Z})$.

Consider the following diagram-keep in mind that we need to distinguish among similar-looking groups $P S L(2, \mathbb{Z}), P S L(2, \mathbb{R})$, etc.:

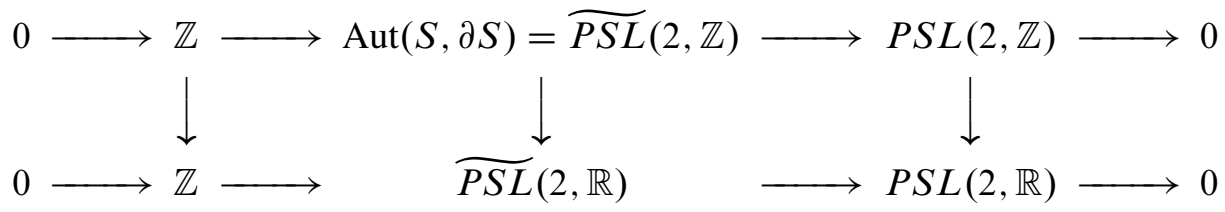

Theorem 2.1 implies the following:

Corollary 3.1 $\delta \Phi=-12 \delta($ rot $)$ as 2 -cochains on $\operatorname{PSL}(2, \mathbb{Z})$.

In other words, two seemingly different quasi-morphisms - the translation number for $\widehat{\text { Homeo }}_{+}\left(S^{1}\right)$ and the Rademacher function-have essentially the same coboundary. Hence, we can keep track of the value of one quasi-morphism through the value of the other quasi-morphism, although the functions are far from identical.

\subsection{The Maslov index}

In this subsection, we define the Maslov index. There are various definitions of the Maslov index in the literature, and our $\mu\left(\gamma, \Lambda_{0}\right)$ is identical to that of Robbin and Salamon in [14].

Consider the symplectic vector space $\left(\mathbb{R}^{2 n}=\mathbb{R}^{n} \times \mathbb{R}^{n}, \omega\right)$, with coordinates $\mathbf{x}=$ $\left(x_{1}, \ldots, x_{n}\right)$ for the first $\mathbb{R}^{n}$ and $\mathbf{y}=\left(y_{1}, \ldots, y_{n}\right)$ for the second $\mathbb{R}^{n}$, and symplectic form $\omega=\sum_{i=1}^{n} d x_{i} \wedge d y_{i}$. Consider the Lagrangian subspace $\Lambda_{0}=\{\mathbf{y}=0\}$. Let $\mathcal{L}$ be the Lagrangian Grassmannian of $\left(\mathbb{R}^{2 n}, \omega\right)$, ie, the set of Lagrangian subspaces of $\mathbb{R}^{2 n}$. Also let $\mathcal{L}_{\Lambda_{0}}$ be the Maslov cycle of $\Lambda_{0}$, namely the set of $\Lambda \in \mathcal{L}$ with $\Lambda_{0} \cap \Lambda \neq\{0\}$. 
If $\Lambda \in \mathcal{L}$ is transverse to $\Lambda_{0}$, then there exists an element of the symplectic group $\operatorname{Sp}(2 n, \mathbb{R})$ which sends $\Lambda_{0}$ to itself and $\Lambda$ to $\Lambda_{0}^{\prime}=\{\mathbf{x}=0\}$. (Proof: If $v_{1}, \ldots, v_{n}$ is a basis for $\Lambda_{0}$ and $w_{1}, \ldots, w_{n}$ is a basis for $\Lambda$, then, with respect to $v_{1}, \ldots, v_{n}$ and $w_{1}, \ldots, w_{n}$, the symplectic form can be written as

$$
\left(\begin{array}{cc}
0 & A \\
-A^{T} & 0
\end{array}\right)
$$

for some nonsingular $n \times n$ matrix $A$. Since we are allowed to change bases of $\Lambda_{0}$ and $\Lambda$ (separately), we are looking to solve:

$$
\left(\begin{array}{cc}
B^{T} & 0 \\
0 & C^{T}
\end{array}\right)\left(\begin{array}{cc}
0 & A \\
-A^{T} & 0
\end{array}\right)\left(\begin{array}{ll}
B & 0 \\
0 & C
\end{array}\right)=\left(\begin{array}{cc}
0 & B^{T} A C \\
-C^{T} A^{T} B & 0
\end{array}\right)=\left(\begin{array}{cc}
0 & I \\
-I & 0
\end{array}\right) .
$$

Such $B, C$ can easily be found.)

Now consider the neighborhood $U=\{\mathbf{y}=A \mathbf{x} \mid A$ symmetric $n \times n$ matrix $\}$ of $\Lambda_{0} \in \mathcal{L}$. (Proof: Any $n$-plane which is sufficiently close to $\mathbf{y}=0$ is graphical of form $\mathbf{y}=A \mathbf{x}$. We can check that the Lagrangian condition implies that $A=A^{T}$.) We observe that $U$ depends on the choice of complementary Lagrangian subspaces $\Lambda_{0}$ and $\Lambda_{0}^{\prime}$, and will often be written as $U\left(\Lambda_{0}, \Lambda_{0}^{\prime}\right)$. It is easy to see that $U$ is contractible.

A useful operation which allows us to cover all of $\mathcal{L}$ with open sets of type $U\left(\Lambda_{0}, \Lambda_{0}^{\prime}\right)$, is the symplectic shear

$$
\left(\begin{array}{ll}
I & A \\
0 & I
\end{array}\right) \in \operatorname{Sp}(2 n, \mathbb{R})
$$

where $A$ is a symmetric $n \times n$ matrix. The shear sends $\Lambda_{0}$ to itself and $\{\mathbf{x}=0\}$ to $\{\mathbf{x}=A \mathbf{y}\}$. If $A$ is invertible, then the Lagrangian subspace $\left\{(A \mathbf{y}, \mathbf{y}) \mid \mathbf{y} \in \mathbb{R}^{n}\right\}=$ $\left\{\left(\mathbf{x}, A^{-1} \mathbf{x}\right) \mid \mathbf{x} \in \mathbb{R}^{n}\right\}$ is in $U\left(\Lambda_{0},\{\mathbf{x}=0\}\right)$. By ranging $\Lambda_{0}^{\prime}$ over all the Lagrangian subspaces transverse to $\Lambda_{0}$, the collection of such $U\left(\Lambda_{0}, \Lambda_{0}^{\prime}\right)$ covers $\mathcal{L}$.

We can now define the Maslov index of a path $\gamma:[0,1] \rightarrow \mathcal{L}$, with respect to a fixed Lagrangian $\Lambda_{0}$. Subdivide $[0,1]$ into $0=t_{0}<t_{1}<\cdots<t_{k}=1$, so that each $\left.\gamma\right|_{\left[t_{i}, t_{i+1}\right]}$ lies in some $U\left(\Lambda_{0}, \Lambda_{0}^{\prime}\right)$. Suppose $\gamma\left(t_{i}\right)$ is given by $\left\{\mathbf{y}=A\left(t_{i}\right) \mathbf{x}\right\}$ and $\gamma\left(t_{i+1}\right)$ by $\left\{\mathbf{y}=A\left(t_{i+1}\right) \mathbf{x}\right\}$. Then let

$$
\mu\left(\left.\gamma\right|_{\left[t_{i}, t_{i+1}\right]}, \Lambda_{0}\right)=\frac{1}{2} \operatorname{sign}\left(A\left(t_{i+1}\right)\right)-\frac{1}{2} \operatorname{sign}\left(A\left(t_{i}\right)\right) .
$$

Here sign denotes the signature of the symmetric matrix. (The signature of a symmetric bilinear form is the dimension of the maximal positive definite subspace minus the 
dimension of the maximal negative definite subspace.) We then define

$$
\mu\left(\gamma, \Lambda_{0}\right) \stackrel{\text { def }}{=} \sum_{i=0}^{k-1} \mu\left(\left.\gamma\right|_{\left[t_{i}, t_{i+1}\right]}, \Lambda_{0}\right) .
$$

By Theorem 2.3 of [14], this $\mu$ is well-defined, invariant under homotopies fixing endpoints, and is natural, ie, $\mu(\Psi(\gamma), \Psi(\Lambda))=\mu(\gamma, \Lambda)$, where $\Psi \in \operatorname{Sp}(2 n, \mathbb{R})$. Moreover, if $\gamma$ does not intersect the Maslov cycle $\mathcal{L}_{\Lambda_{0}}$, then $\mu\left(\gamma, \Lambda_{0}\right)=0$. In the special case that $\gamma$ is a closed loop, $\mu\left(\gamma, \Lambda_{0}\right)$ is independent of the choice of $\Lambda_{0}$.

Next, given $L_{1}, L_{2}, L_{3} \in \mathcal{L}$, we define the ternary index $I\left(L_{1}, L_{2}, L_{3}\right)$. Consider the symmetric bilinear form $Q$ on $\left(L_{1}+L_{2}\right) \cap L_{3}$ defined by $Q(v, w)=\omega\left(v_{2}, w\right)$, where $v \in\left(L_{1}+L_{2}\right) \cap L_{3}$ is written as $v_{1}+v_{2}$, with $v_{1} \in L_{1}, v_{2} \in L_{2}$. Then $I\left(L_{1}, L_{2}, L_{3}\right)$ is the signature of $Q$.

It is not difficult to see that $I\left(L_{1}, L_{2}, L_{3}\right)$ has the following equivalent definition: Consider the subspace $V \subset L_{1} \oplus L_{2} \oplus L_{3}$, consisting of triples $\left(v_{1}, v_{2}, v_{3}\right), v_{i} \in L_{i}$, with $v_{1}+v_{2}+v_{3}=0$. Define the quadratic form $Q^{\prime}: V \times V \rightarrow \mathbb{R}$ by:

$$
\begin{aligned}
Q^{\prime}\left(\left(v_{1}, v_{2}, v_{3}\right),\left(w_{1}, w_{2}, w_{3}\right)\right) & =\omega\left(v_{1}, w_{3}\right)=\omega\left(v_{2}, w_{1}\right)=\omega\left(v_{3}, w_{2}\right) \\
& =-\omega\left(v_{3}, w_{1}\right)=-\omega\left(v_{1}, w_{2}\right)=-\omega\left(v_{2}, w_{3}\right) .
\end{aligned}
$$

Then $I\left(L_{1}, L_{2}, L_{3}\right)$ is also the signature of $Q^{\prime}$.

Now, given $L_{1}, L_{2}, L_{3} \in \mathcal{L}$, let $\gamma_{12}$ be a path in $\mathcal{L}$ from $L_{1}$ to $L_{2}, \gamma_{23}$ be a path in $\mathcal{L}$ from $L_{2}$ to $L_{3}$, and let $\gamma_{13}$ be the concatenation $\gamma_{12} \gamma_{23}$. Also let $\gamma_{i j}=\gamma_{j i}^{-1}$. We then have the following:

Lemma 3.2 $I\left(L_{1}, L_{2}, L_{3}\right)=2\left(\mu\left(\gamma_{12}, L_{1}\right)+\mu\left(\gamma_{23}, L_{2}\right)+\mu\left(\gamma_{31}, L_{3}\right)\right)$.

Proof Suppose $L_{1}, L_{2}, L_{3}$ are mutually transverse. Without loss of generality, we may take $L_{1}=\{\mathbf{y}=0\}, L_{2}=\{\mathbf{x}=0\}$, and $L_{3}=\{\mathbf{y}=A \mathbf{x}\}$, where $A$ is symmetric and nonsingular.

Since the right-hand side of the equation in the lemma does not depend on the choice of paths, provided the endpoints remain the same, there is no loss of generality in proving the lemma for a convenient choice of paths. (As remarked earlier, if $\gamma$ is a loop, then $\mu(\gamma, L)$ does not depend on the choice of $L \in \mathcal{L}$.) Define $\gamma_{13}:[0,1] \rightarrow \mathcal{L}$ by $t \mapsto\{\mathbf{y}=t A \mathbf{x}\}, \gamma_{23}:[0,1] \rightarrow \mathcal{L}$ by $t \mapsto\left\{\mathbf{x}=t A^{-1} \mathbf{y}\right\}$, and $\gamma_{12}=\gamma_{13} \gamma_{32}$. One 
easily computes from Equation (3.3.1) that

$$
\begin{aligned}
& \mu\left(\gamma_{12}, L_{1}\right)=\frac{1}{2} \operatorname{sign}(A), \\
& \mu\left(\gamma_{23}, L_{2}\right)=-\frac{1}{2} \operatorname{sign}\left(A^{-1}\right)=-\frac{1}{2} \operatorname{sign}(A), \\
& \mu\left(\gamma_{31}, L_{3}\right)=-\frac{1}{2} \operatorname{sign}(A) .
\end{aligned}
$$

Therefore, the right-hand side of the equation in the lemma equals $-\operatorname{sign}(A)$.

On the other hand, $\left(L_{1}+L_{2}\right) \cap L_{3}=L_{3}$ in our case, and

$$
Q\left((\mathbf{x}, A \mathbf{x}),\left(\mathbf{x}^{\prime}, A \mathbf{x}^{\prime}\right)\right)=\omega\left((0, A \mathbf{x}),\left(\mathbf{x}^{\prime}, 0\right)\right)=-\mathbf{x}^{T} A^{T} \mathbf{x}^{\prime} .
$$

Thus, $I\left(L_{1}, L_{2}, L_{3}\right)=-\operatorname{sign}(A)$ as well.

The general case is more involved, and we only sketch the idea. First, we normalize $L_{1}, L_{2}, L_{3}$ (this requires some work), and then use the additivity formula from [14]. Let $V^{\prime}$ be a Lagrangian of standard symplectic $\mathbb{R}^{2 n^{\prime}}$ and $V^{\prime \prime}$ be a Lagrangian of $\mathbb{R}^{2 n^{\prime \prime}}$. Also let $\gamma^{\prime}$ be a path in $\mathcal{L}\left(\mathbb{R}^{2 n^{\prime}}\right)$ and $\gamma^{\prime \prime}$ be a path in $\mathcal{L}\left(\mathbb{R}^{2 n^{\prime \prime}}\right)$. Then the additivity formula says the following:

$$
\mu\left(\gamma^{\prime} \oplus \gamma^{\prime \prime}, V^{\prime} \oplus V^{\prime \prime}\right)=\mu\left(\gamma^{\prime}, V^{\prime}\right)+\mu\left(\gamma^{\prime \prime}, V^{\prime \prime}\right) .
$$

We then reduce to the above calculation where $L_{1}, L_{2}, L_{3}$ are mutually transverse. $\square$

\subsection{The signature}

To generalize the results we obtained for the punctured torus to the braid group $B_{n}$ on $n$ strands, we use the signature. The signature has the useful property of remaining invariant under stabilization, whereas the linking number increases under stabilization.

Define the signature function on $B_{n}$ as follows: Given a braid $\alpha$, let $\widehat{\alpha}$ denote the braid closure inside $S^{3}$, and $\operatorname{sign}(\widehat{\alpha})$ be the signature of the (symmetrized) Seifert pairing. It is not difficult to see that the signature is a quasi-morphism on $B_{n}$.

We can view $B_{2 n+1}$ as the hyperelliptic mapping class group of a once-punctured surface $\Sigma_{n}$, where $n$ is the genus, and $B_{2 n}$ as the hyperelliptic mapping class group of a twice-punctured surface $\Sigma_{n-1}$, where $n-1$ is the genus. (Here by the genus we mean the genus of the closed surface obtained by adding disks.) For $B_{2 n+1}$, let $\mathcal{B}_{-1}$ be the map $B_{2 n+1} \rightarrow S p(2 n, \mathbb{R})$, which is the action on the symplectic vector space $H_{1}\left(\Sigma_{n}, \partial \Sigma_{n} ; \mathbb{R}\right)$ (with symplectic form the intersection pairing). For $B_{2 n}$, the intersection pairing is degenerate, so we take the standard embedding of $B_{2 n}$ into $B_{2 n+1}$ by adding a trivial strand; from now on we assume that our braid groups have 
an odd number of stands. The strange notation $\mathcal{B}_{-1}$ comes from the fact that the homology representation is the Burau representation specialized at -1 . Note that for $n=1$ it is the same as the map $B_{3} \rightarrow S L(2, \mathbb{Z})$ which appeared in Section 2.

Next we define the Meyer cocycle Meyer $\left(g_{1}, g_{2}\right)$, where $g_{1}, g_{2} \in S p(2 n, \mathbb{R})$. (Here we are thinking of $H_{1}\left(\Sigma_{n}, \partial \Sigma_{n} ; \mathbb{R}\right)$ as a symplectic vector space of dimension $2 n$.) Consider the symplectic vector space $\left(\mathbb{R}^{2 n} \times \mathbb{R}^{2 n}, \omega \oplus-\omega\right)$. Let $\tilde{g}_{1}$ be a path in $S p(2 n, \mathbb{R})$ from id to $g_{1}$ and $\widetilde{g}_{2}$ be a path from id to $g_{2}$. Also let $\widetilde{g_{1} g_{2}}(t)=$ $\tilde{g}_{1}(t) \widetilde{g}_{2}(t), t \in[0,1]$; this is homotopic to the path $\widetilde{g}_{1}(t)$, followed by the path $g_{1} \cdot\left(\widetilde{g}_{2}(t)\right)$. Now let $\operatorname{Graph}(h)$ be the graph of $h \in S p(2 n, \mathbb{R})$, ie, it is the Lagrangian of $\mathbb{R}^{2 n} \oplus \mathbb{R}^{2 n}$ consisting of vectors $(v, h(v))$; if $\tilde{h}$ is a path in $\operatorname{Sp}(2 n, \mathbb{R}), \operatorname{Graph}(\tilde{h})$ is a path of Lagrangians $\operatorname{Graph}(\tilde{h}(t)), t \in[0,1]$. Then we set:

$$
\begin{aligned}
\operatorname{Meyer}\left(g_{1}, g_{2}\right) \stackrel{\text { def }}{=} & I\left(\operatorname{Graph}(\mathrm{id}), \operatorname{Graph}\left(g_{1}\right), \operatorname{Graph}\left(g_{1} g_{2}\right)\right) \\
= & 2\left(\mu\left(\operatorname{Graph}\left(\widetilde{g}_{1}\right), \operatorname{Graph}(\mathrm{id})\right)+\mu\left(\operatorname{Graph}\left(\widetilde{g}_{2}\right), \operatorname{Graph}(\mathrm{id})\right)\right. \\
& \left.-\mu\left(\operatorname{Graph}\left(\widetilde{g_{1} g_{2}}\right), \operatorname{Graph}(\mathrm{id})\right)\right) .
\end{aligned}
$$

(It is not hard to verify that if $\gamma:[0,1] \rightarrow \mathcal{L}$ is a path of Lagrangians, then $\mu(\gamma, \gamma(0))=$ $-\mu\left(\gamma^{-1}, \gamma^{-1}(0)\right)$, where $\gamma^{-1}(t)=\gamma(1-t)$. We also used the fact that $\mu(\gamma, \Lambda)=$ $\mu(\Psi(\gamma), \Psi(\Lambda))$.)

In [5] it is proven that:

$$
\operatorname{sign}(\widehat{\alpha \beta})=\operatorname{sign}(\widehat{\alpha})+\operatorname{sign}(\widehat{\beta})-\operatorname{Meyer}\left(\mathcal{B}_{-1}(\alpha), \mathcal{B}_{-1}(\beta)\right) .
$$

There are two quasi-morphisms whose coboundary is the Meyer cocycle: on $B_{2 n+1}$ there is the signature, and on $\widetilde{S p}(2 n, \mathbb{R})$ there is the Maslov index. Here $\widetilde{S p}(2 n, \mathbb{R})$ is the universal cover of $\operatorname{Sp}(2 n, \mathbb{R})$. In order to relate the two, we first observe the following:

Lemma 3.3 The homomorphism $\mathcal{B}_{-1}: B_{2 n+1} \rightarrow S p(2 n, \mathbb{R})$ can be lifted to a homomorphism $\widetilde{\mathcal{B}}_{-1}: B_{2 n+1} \rightarrow \widetilde{S p}(2 n, \mathbb{R})$.

Proof Let $C_{1}, \ldots, C_{2 n}$ be oriented nonseparating closed curves on $\Sigma_{n}$ so that (i) $\sigma_{i}$ corresponds to a positive Dehn twist about $C_{i}$ and (ii) the intersection pairing $\omega\left(C_{i}, C_{j}\right)$ is $\delta_{i+1, j}-\delta_{i-1, j}$. (Here $\delta_{i, j}$ is 0 if $i \neq j$ and 1 if $\left.i=j.\right)$

Let $A_{1}$ be the $2 \times 2$ matrix

$$
\left(\begin{array}{ll}
1 & 1 \\
0 & 1
\end{array}\right)
$$


and let $A_{2}$ be the $3 \times 3$ matrix

$$
\left(\begin{array}{ccc}
1 & 0 & 0 \\
-1 & 1 & 1 \\
0 & 0 & 1
\end{array}\right)
$$

Then $\mathcal{B}_{-1}\left(\sigma_{1}\right)=\operatorname{diag}\left(A_{1}, 1, \ldots, 1\right)$ (ie, the matrix with the given entries along the diagonal and zeros everywhere else). We also have $\mathcal{B}_{-1}\left(\sigma_{2}\right)=\operatorname{diag}\left(A_{2}, 1, \ldots, 1\right), \ldots$, $\mathcal{B}_{-1}\left(\sigma_{2 n-1}\right)=\operatorname{diag}\left(1, \ldots, 1, A_{2}\right)$, and $\mathcal{B}_{-1}\left(\sigma_{2 n}\right)=\operatorname{diag}\left(1, \ldots, 1, A_{1}\right)$.

To lift to $\widetilde{S p}(2 n, \mathbb{R})$, we replace $A_{1}$ by

$$
A_{1}(t)=\left(\begin{array}{ll}
1 & t \\
0 & 1
\end{array}\right)
$$

and $A_{2}$ by

$$
A_{2}(t)=\left(\begin{array}{ccc}
1 & 0 & 0 \\
-t & 1 & t \\
0 & 0 & 1
\end{array}\right),
$$

where $t \in[0,1]$. (We do this for all the $\mathcal{B}_{-1}\left(\sigma_{i}\right)$.) To verify that this indeed gives a lift $\widetilde{\mathcal{B}}_{-1}: B_{n} \rightarrow \widetilde{S p}(2 n, \mathbb{R})$, we need to check the braid relations.

If $|i-j| \geq 2$, then $\sigma_{i} \sigma_{j}=\sigma_{j} \sigma_{i}$, and to verify that $\widetilde{\mathcal{B}}_{-1}\left(\sigma_{i}\right) \widetilde{\mathcal{B}}_{-1}\left(\sigma_{j}\right)=\widetilde{\mathcal{B}}_{-1}\left(\sigma_{j}\right) \widetilde{\mathcal{B}}_{-1}\left(\sigma_{i}\right)$ it suffices to check that $\operatorname{diag}\left(A_{2}(t), 1,1\right)$ and $\operatorname{diag}\left(1,1, A_{2}(t)\right)$ commute. This is an easy calculation. (The cases $i=1, j=3$ and $i=2 n-2, j=n$, when $\operatorname{diag}\left(A_{1}(t), 1,1\right)$ and $\operatorname{diag}\left(1,1, A_{1}(t)\right)$ are involved, are easier.)

We also verify that

$$
\widetilde{\mathcal{B}}_{-1}\left(\sigma_{i}\right) \widetilde{\mathcal{B}}_{-1}\left(\sigma_{i+1}\right) \widetilde{\mathcal{B}}_{-1}\left(\sigma_{i}\right)=\widetilde{\mathcal{B}}_{-1}\left(\sigma_{i+1}\right) \tilde{\mathcal{B}}_{-1}\left(\sigma_{i}\right) \tilde{\mathcal{B}}_{-1}\left(\sigma_{i+1}\right) .
$$

Computing both sides, we require:

$$
\left(\begin{array}{cccc}
1 & 0 & 0 & 0 \\
-2 t+t^{3} & 1-t^{2} & 2 t-t^{3} & t^{2} \\
t^{2} & -t & 1-t^{2} & t \\
0 & 0 & 0 & 1
\end{array}\right) \quad \text { and } \quad\left(\begin{array}{cccc}
1 & 0 & 0 & 0 \\
-t & 1-t^{2} & t & t^{2} \\
t^{2} & -2 t+t^{3} & 1-t^{2} & 2 t-t^{3} \\
0 & 0 & 0 & 1
\end{array}\right)
$$

to be homotopic as paths. Since $-t$ and $-2 t+t^{3}$ are both negative for $t \in(0,1]$, we can take $a(s, t)=(1-s)\left(-2 t+t^{3}\right)+s(-t)$ and $b(s, t)=(-t)\left(-2 t+t^{3}\right) / a(s, t)$. Now

$$
\left(\begin{array}{cccc}
1 & 0 & 0 & 0 \\
a(s, t) & 1-t^{2} & -a(s, t) & t^{2} \\
t^{2} & b(s, t) & 1-t^{2} & -b(s, t) \\
0 & 0 & 0 & 1
\end{array}\right)
$$


is a homotopy of paths in $\operatorname{Sp}(2 n, \mathbb{R})$ which takes the left-hand matrix in Equation (3.4.2) to the right-hand one in Equation (3.4.2).

The following is a relatively simple computation, once the definitions are sorted out:

Lemma 3.4 $\mu(\operatorname{Graph}(\tilde{h}), \operatorname{Graph}(\mathrm{id}))=1 / 2$ if $h \in \operatorname{Sp}(2 n, \mathbb{R})$ is a positive Dehn twist about a nonseparating curve.

Proof We first reduce to the case where $n=1$, the symplectic form on $\mathbb{R}^{2} \oplus \mathbb{R}^{2}$ (with coordinates $\left.(x, y)=\left(\left(x_{1}, x_{2}\right),\left(y_{1}, y_{2}\right)\right)\right)$ is $\omega=d x_{1} \wedge d x_{2}-d y_{1} \wedge d y_{2}$, and

$$
h=\left(\begin{array}{ll}
1 & 1 \\
0 & 1
\end{array}\right) .
$$

Indeed, Graph(id) is the set $\left\{(x, x) \mid x \in \mathbb{R}^{2 n}\right\}=\left\{(g x, g x) \mid x \in \mathbb{R}^{2 n}\right\}$ and $\operatorname{Graph}(h)$ is the set $\left\{(x, h x) \mid x \in \mathbb{R}^{2 n}\right\}=\left\{(g x, h g x) \mid x \in \mathbb{R}^{2 n}\right\}$, if $g$ is a nonsingular $2 n \times 2 n$ matrix. Now, apply $\left(g^{-1}, g^{-1}\right)$ to both $\operatorname{Graph}(\mathrm{id})$ and $\operatorname{Graph}(h)$, where $g \in \operatorname{Sp}(2 n, \mathbb{R})$. This gives us Graph(id) and Graph $\left(g^{-1} h g\right)$. Hence, by conjugating, we may assume that $h$ is as above, since $\mu$ is invariant under the action of the symplectic group.

The graph of id, which we write as $L_{0}$, is $\mathbb{R}\left\{v_{1}=(1,0,1,0), v_{2}=(0,1,0,1)\right\}$. A complementary Lagrangian subspace to $L_{0}$ is $L_{0}^{\prime}=\mathbb{R}\left\{w_{1}=(0,0,0,-1), w_{2}=\right.$ $(-1,0,0,0)\}$. (Here $\omega\left(v_{i}, w_{j}\right)=\delta_{i, j}$.) The graph of $h$ is spanned by $v_{1}=(1,0,1,0)$ and $v_{1}+v_{2}+w_{2}=(0,1,1,1)$, or, equivalently, by $v_{1}$ and $v_{2}+w_{2}$. Hence

$$
\mu(\operatorname{Graph}(\tilde{h}), \operatorname{Graph}(\mathrm{id}))=\frac{1}{2} \operatorname{sign}\left(\begin{array}{ll}
0 & 0 \\
0 & 1
\end{array}\right)=\frac{1}{2} .
$$

This proves the lemma.

We now state the main theorem of this section:

Theorem 3.5 Let $\gamma$ be an element of $B_{2 n+1}$, or equivalently, an element of a hyperelliptic mapping class group $\operatorname{Hyp} \operatorname{Aut}\left(\Sigma_{n}, \partial \Sigma_{n}\right)$. Then

$$
\operatorname{sign}(\hat{\gamma})=-\operatorname{lk}(\gamma)+2 \mu\left(\operatorname{Graph}\left(\tilde{\mathcal{B}}_{-1}(\gamma)\right), \operatorname{Graph}(\mathrm{id})\right) .
$$

Since $\operatorname{lk}(\gamma) \geq 0$ if $\gamma$ is quasipositive, we have the following:

Corollary 3.6 If $\operatorname{sign}(\hat{\gamma})>2 \mu\left(\operatorname{Graph}\left(\tilde{\mathcal{B}}_{-1}(\gamma)\right)\right.$, Graph(id) $)$, then $\gamma$ cannot be quasipositive in $B_{2 n+1}$. Equivalently, $\gamma$ is not a product of positive Dehn twists in $\operatorname{Hyp} \operatorname{Aut}\left(\Sigma_{n}, \partial \Sigma_{n}\right)$. 
Proof Let $\gamma$ be an element of $B_{2 n+1}$. Then $\gamma$ can be written as $\gamma=\gamma_{1} \ldots \gamma_{k}$, where $\gamma_{i}$ are all conjugates of a standard half-twist or its inverse. Let $g_{i}=\mathcal{B}_{-1}\left(\gamma_{i}\right) \in$ $S p(2 n, \mathbb{R})$, and let $\widetilde{g}_{i}=\widetilde{\mathcal{B}}_{-1}\left(\gamma_{i}\right) \in \widetilde{S p}(2 n, \mathbb{R})$ be a path from id to $g_{i}$ in $\operatorname{Sp}(2 n, \mathbb{R})$. By repeatedly using Equation (3.4.1) and observing that $\operatorname{sign}\left(\widehat{\gamma}_{i}\right)=0$ (since the Seifert surface is a disk), we have:

$$
\begin{aligned}
\operatorname{sign}\left(\widehat{\gamma_{1} \ldots \gamma_{k}}\right) & =\operatorname{sign}\left(\widehat{\gamma}_{1}\right)+\operatorname{sign}\left(\widehat{\gamma_{2} \ldots \gamma_{k}}\right)-\operatorname{Meyer}\left(\mathcal{B}_{-1}\left(\gamma_{1}\right), \mathcal{B}_{-1}\left(\gamma_{2} \ldots \gamma_{k}\right)\right) \\
& =\operatorname{sign}\left(\widehat{\gamma_{2} \ldots \gamma_{k}}\right)-\operatorname{Meyer}\left(\mathcal{B}_{-1}\left(\gamma_{1}\right), \mathcal{B}_{-1}\left(\gamma_{2} \ldots \gamma_{k}\right)\right) \\
& =-\sum_{i=1}^{k-1} \operatorname{Meyer}\left(g_{i}, g_{i+1} \ldots g_{k}\right) .
\end{aligned}
$$

Next, applying Lemma 3.4, we have:

$$
\begin{aligned}
& \operatorname{sign}\left(\widehat{\gamma_{1} \ldots \gamma_{k}}\right)=-2 \sum_{i=1}^{k-1}\{ \mu\left(\operatorname{Graph}\left(\widetilde{g}_{i}\right), \operatorname{Graph}(\mathrm{id})\right) \\
&+\mu\left(\operatorname{Graph}\left(\widetilde{g_{i+1} g_{k}}\right), \operatorname{Graph}(\mathrm{id})\right) \\
&\left.-\mu\left(\operatorname{Graph}\left(\widetilde{g_{i} \ldots g_{k}}\right), \operatorname{Graph}(\mathrm{id})\right)\right\} \\
&=-2 \sum_{i=1}^{k-1}\left\{ \pm \frac{1}{2}+\mu\left(\operatorname{Graph}\left(\widehat{g_{i+1} \ldots g_{k}}\right), \operatorname{Graph}(\mathrm{id})\right)\right. \\
&\left.-\mu\left(\operatorname{Graph}\left(\widetilde{g_{i} \ldots g_{k}}\right), \operatorname{Graph}(\mathrm{id})\right)\right\} \\
&=-1 \mathrm{k}(\gamma)+2 \mu\left(\operatorname{Graph}\left(\widetilde{g_{1} \ldots g_{k}}\right), \operatorname{Graph}(\mathrm{id})\right) .
\end{aligned}
$$

Here we have $\pm 1 / 2$ depending on whether we have a positive or negative Dehn twist. If $\gamma$ is quasipositive, $\operatorname{lk}(\gamma) \geq 0$. So $\operatorname{sign}(\gamma) \leq 2 \mu\left(\operatorname{Graph}\left(\widetilde{g_{1} \ldots g_{k}}\right)\right.$, Graph(id) $)$.

Remark In [5], Gambaudo and Ghys prove that, for the "generic element" $\gamma \in B_{3}$,

$$
\operatorname{sign}(\hat{\gamma})+\frac{2}{3} \operatorname{lk}(\gamma)=-\frac{1}{3} \Phi\left(\mathcal{B}_{-1}(\gamma)\right) .
$$

For example, if $\gamma$ is generic if it is pseudo-Anosov. Combining with Theorem 2.1, we have:

$$
\operatorname{sign}(\hat{\gamma})=-\operatorname{lk}(\gamma)+4 \operatorname{rot}(\gamma),
$$

for such $\gamma$. This is consistent with Theorem 3.5.

Remark Gambaudo and Ghys also have a formula analogous to Equation (3.4.1) for the $\omega$-signatures. Presumably our Theorem 3.5 can be generalized to $\omega$-signatures as well. 


\section{Characterization of (weak) symplectic fillability}

In this section we prove Theorem 1.2. The starting point is the following special case of a theorem of Roberts [15; 16], generalizing work of Hatcher [8].

Theorem 4.1 (Roberts) Assume the surface $S$ has one boundary component and $h$ is a diffeomorphism that restricts to the identity on the boundary. If $h$ is isotopic to a pseudo-Anosov homeomorphism $\psi$ and the fractional Dehn twist coefficient of $h$ is $c$, then $M=(S, h)$ carries a taut foliation transverse to the binding if $c \geq 1$.

This theorem (not stated in this form by Roberts) follows from a more general result of Roberts which is stated below as Theorem 4.2. To explain how Theorem 4.1 follows from Theorem 4.2, we start by comparing the notation and coordinates used by Roberts to our own.

Let $S$ be a hyperbolic surface with one boundary component, $\psi$ be the pseudo-Anosov representative of $h$, and $c$ be the fractional Dehn twist coefficient. Denote by $N$ the mapping torus of $\psi$, ie, $N:=(S \times[0,1]) /(x, 1) \sim(\psi(x), 0)$ for $x \in S$.

Roberts gives an oriented identification $\partial N \simeq \mathbb{R}^{2} / \mathbb{Z}^{2}$ by choosing closed curves $\lambda, \mu$ so that $\lambda$ has slope 0 and $\mu$ has slope $\infty$. (See Section 3 of [16].) Here we choose orientations to agree with the usual conventions for a knot complement. We will now describe the curves $\lambda$ and $\mu$. Let $\lambda=\partial(S \times\{0\})$. Define $\gamma$ to be one component of the suspension of the periodic points of $\left.\psi\right|_{\partial S}$. If there are $n$ prongs, then there are $2 n$ periodic points, $n$ of which are attracting and $n$ of which are expanding. Observe that the geometric intersection number $\#(\gamma \cap(S \times\{0\}))$ divides $n$ and equals $n$ if the suspension (of only the attracting points) is connected. Now we define $\mu$ to be the essential closed curve on $\partial N$ which has the minimal $\#(\mu \cap \gamma)$ amongst all closed curves on $\partial N$ which form an integral basis of $H_{1}(\partial N ; \mathbb{Z})$ with $\lambda$. The choice of $\mu$ is not unique if $\#(\gamma \cap \lambda)=2$; there are two choices which minimize $\#(\mu \cap \gamma)$. In that case we choose $\mu$ so that $\operatorname{slope}(\gamma)=+2$.

We now state Theorem 4.7 of [16]:

Theorem 4.2 (Roberts) Suppose $S$ has one boundary component, $\psi$ is a pseudoAnosov map and $N=(S \times[0,1]) /(x, 1) \sim(\psi(x), 0)$. Then one of the following holds:

(1) $\gamma$ has slope infinity and $N$ contains taut foliations realizing all boundary slopes in $(-\infty, \infty)$.

(2) $\gamma$ has positive slope and $N$ contains taut foliations realizing all boundary slopes in $(-\infty, 1)$. 
(3) $\gamma$ has negative slope and $N$ contains taut foliations realizing all boundary slopes in $(-1, \infty)$.

Here the slope is measured with respect to the identification $\partial N \simeq \mathbb{R}^{2} / \mathbb{Z}^{2}$ given by the basis $(\lambda, \mu)$ defined above, and "realizing" a boundary slope means the restriction of the taut foliation to $\partial N$ is a linear foliation with the given boundary slope.

Proof that Theorem 4.2 implies Theorem 4.1 Suppose $h \in \operatorname{Aut}(S, \partial S)$, $\psi$ is its pseudo-Anosov representative, and $c=p / q$ is the corresponding fractional Dehn twist coefficient. Assume $p, q$ are relatively prime positive integers. If the closed manifold $M=(S, h)$ is obtained by Dehn filling $N$ along the closed curve $v$ on $\partial N$, then $\gamma=p \lambda+q v$ in $H_{1}(\partial N ; \mathbb{Z})$. We also have $\mu=v+k \lambda$, where $k$ is an integer chosen to minimize $|\gamma \cdot \mu|=|(p \lambda+q v) \cdot(v+k \lambda)|=|p-k q|$. When there is a tie, ie, both $p-k q= \pm p / 2$ are possible, the tie is broken by choosing $p / 2$. In the cases below, the slope will be computed relative to the basis $(\lambda, \mu)$. We compute that $\operatorname{slope}(\nu)=(\lambda \cdot v) /(\nu \cdot \mu)=-1 / k$ and slope $(\gamma)=q / p-k q$.

(1) $c=p / q$ is an integer $\geq 1$. It follows that $p-k q=0$ and $k \geq 1$. Therefore, $\operatorname{slope}(\gamma)=\infty$ and $\operatorname{slope}(v)=-1 / k \in[-1,0) \subset(-\infty, \infty)$.

(2) $p / q>1$ is not an integer and $k$ satisfies $0<p-k q \leq p / 2$. It follows that slope $(\gamma)>0, k \geq 1$, and therefore slope $(v)=-1 / k \in[-1,0) \subset(-\infty, 1)$.

(3) $p / q>1$ is not an integer and $k$ satisfies $-p / 2<p-k q<0$. It follows that slope $(\gamma)<0, k \geq 2$, and therefore slope $(v)=-1 / k \in[-1 / 2,0) \subset(-1, \infty)$.

Thus, for all $c \geq 1$, a taut foliation of $N$ can be constructed with boundary slope equal to the slope of the meridian of the solid torus that extends $N$ to $M$. By extending the leaves by meridian disks, we can construct a taut foliation of $M$ transverse to the binding.

If $M=(S, h)$ and $c \geq 1$, let $\mathcal{F}$ be a taut foliation furnished by Theorem 4.1. By the work of Eliashberg-Thurston [2], any taut foliation admits a $C^{0}$-small perturbation into a universally tight and (weakly) symplectically fillable contact structure. We denote a perturbation of $\mathcal{F}$ by $\xi_{\mathcal{F}}$. (Note that, a priori, two perturbations of $\mathcal{F}$ may not even be isotopic.) We will denote by $(S, h)$ the contact structure corresponding to the open book, which is also denoted $(S, h)$.

Theorem 4.3 If $c \geq 1$, then the contact structure $(S, h)$ is isotopic to $\xi_{\mathcal{F}}$ for some taut foliation $\mathcal{F}$.

The proof of Theorem 4.3 will occupy the rest of the section. We first claim the following: 
Lemma 4.4 Let $\psi$ be a pseudo-Anosov representative of $h \in \operatorname{Aut}(S, \partial S)$ with $c \geq 1$ and let $N=(S \times[0,1]) /(x, 1) \sim(\psi(x), 0)$. Then there exists a nonsingular vector field $X$ on $N$ with the following properties:

(1) $X$ is tangent to $\partial N$.

(2) $X$ is positively transverse to $S \times\{t\}$ for all $t \in[0,1]$.

(3) There exists a transversely oriented taut foliation $\mathcal{F}$ on $N$ which is positively transverse to $X$. Moreover, $\mathcal{F}$ can be chosen so that $\mathcal{F} \cap T(\partial N)$ is a nonsingular foliation on $\partial N$ which is foliated by circles of slope $-1 / k$, where $k$ is the positive integer as described in the proof of Theorem 4.2.

Here we are using slope convention used in Theorem 4.2.

Proof This follows from analyzing Roberts' construction (cf Section 2 of [15]) of the foliation $\mathcal{F}$ in Theorem 4.1 and noting that it can be performed in a manner compatible with $X$. Roberts constructs a set $\alpha_{1}, \ldots, \alpha_{m}$ of properly embedded oriented arcs in $S$ with the following properties. Consider $D_{i}=\alpha_{i} \times[(i-1) / m, i / m]$, where $D_{i}$ is oriented so that $(\partial / \partial t, \dot{\alpha})$ form an oriented basis for $T D_{i}$. (Here $t$ is the coordinate for $[0,1]$.) Also write $S_{t}:=S \times\{t\}$. This gives a spine

$$
\Sigma=\left(\bigcup_{i=1}^{m} S_{i / m}\right) \cup\left(\bigcup_{i=1}^{m} D_{i}\right),
$$

which can be modified into a branched surface $\mathcal{B}$ by isotoping $D_{i}$ and smoothing the neighborhood of each intersection $\alpha_{i} \times\{i / m\}$ between $S_{i / m}$ and $D_{i}$ into a branch locus, so that, near the branch locus, a vector field which is positively transverse to $S_{i / m}$ (we may take $\partial / \partial t$ here) is also positively transverse to the new $D_{i}$. The same can be done for each intersection $\alpha_{i} \times\{(i-1) / m\}$ between $S_{(i-1) / m}$ and $D_{i}$.

On each $S \times[(i-1) / m, i / m]$, start with $\partial / \partial t$, which satisfies (1) and (2), and tilt it near $D_{i}$ so that the resulting $X$ becomes positively transverse to $\mathcal{B}$, while still keeping properties (1) and (2). (The other option is to keep $\partial / \partial t$ and smooth the spine into a branched surface so that each $D_{i}$ no longer has any vertical tangencies.) The foliation $\mathcal{F}$ is constructed by first taking a lamination which is fully carried by $\mathcal{B}$ and by extending it to complementary regions which are $I$-bundles. The $I$-fibers can be taken to be tangent to $X$ and hence the foliations on the $I$-bundles transverse to $X$.

Recall that the ambient manifold $M$ can be written as $N \cup\left(S^{1} \times D^{2}\right)$, where the meridian of the solid torus has slope $-1 / k$ on $\partial N$. Here $k$ is the integer in the proof of Theorem 4.2. The foliation $\mathcal{F}$ on $N$ is now extended to all of $M$ (also called $\mathcal{F}$ ) by foliating $S^{1} \times D^{2}$ by meridian disks. 
Lemma 4.5 There exists an isotopy $\phi: S \times[0,1] \rightarrow N$ so that the following hold:

(1) $\phi_{S}(S)$ is properly embedded for all $s \in[0,1]$. Here $\phi_{s}(y) \stackrel{\text { def }}{=} \phi(y, s)$.

(2) $\phi_{0}(S)=S \times\{0\}$.

(3) $\phi_{s}(S)$ is positively transverse to $X$ for all $s$.

(4) $\partial\left(\phi_{1}(S)\right)$ is positively transverse to $\mathcal{F}$.

Here the orientation on $\partial\left(\phi_{S}(S)\right)$ is the one induced from $\phi_{S}(S)$, which in turn is consistent with that of $S \times\{t\}$.

Proof It suffices to show that there is an isotopy $\psi: S^{1} \times[0,1] \rightarrow \partial N$ so that:

(1) $\psi_{s}$ is an embedding for all $s \in[0,1]$.

(2) $\psi_{0}\left(S^{1}\right)=\partial(S \times\{0\})$ (and their orientations agree).

(3) $\psi_{s}\left(S^{1}\right)$ is transverse to $\left.X\right|_{\partial N}$ for all $s$ and $\left(\dot{\psi}_{s}, X\right)$ form an oriented basis of $\partial N$.

(4) $\psi_{1}\left(S^{1}\right)$ is positively transverse to $\mathcal{F}$. Here $\mathcal{F}$ intersects $\partial N$ transversely.

To demonstrate the existence of such an isotopy, we examine the train track $\mathcal{T}=\mathcal{B} \cap \partial N$, where $\mathcal{B}$ is the branched surface constructed in Lemma 4.4. We use standard Euclidean coordinates $(x, y)$ on $\partial N \simeq \mathbb{R}^{2} / \mathbb{Z}^{2}$ (given by Roberts as $(\lambda, \mu)$ ). By construction, $\{y=0\} \subset \mathcal{T}$. Now $\{-\varepsilon \leq y \leq \varepsilon\} \cap \mathcal{T}$ has four branch points, all on $\{y=0\}$. Two of the branches come in from $\{0 \leq y \leq \varepsilon\}$ and the other two come in from $\{-\varepsilon \leq y \leq 0\}$. (See Figure 4.) Since they are coming from a single disk in $N$, the branching directions of the two branches on $\{0 \leq y \leq \varepsilon\}$ are opposite and so are the branching directions of the two branches on $\{-\varepsilon \leq y \leq 0\}$. Here the branching direction at a branch point is the direction in which two branches come together to become one. We will assume that $X=\partial / \partial y$ on $\{-\varepsilon \leq y \leq \varepsilon\}$.

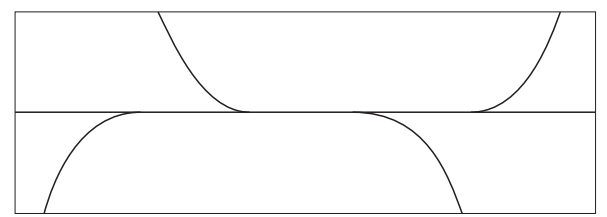

(a)

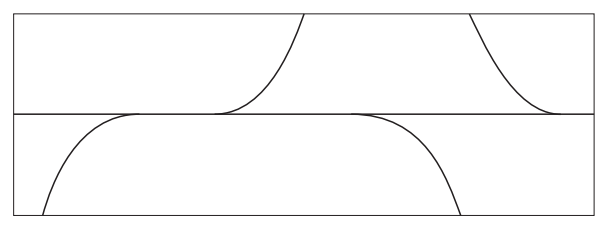

(b)

Figure 4: The train track $\mathcal{T}$ near $y=0$. The bounding box represents $[0,1] \times[-\varepsilon, \varepsilon] \subset \mathbb{R}^{2} / \mathbb{Z}^{2}$. Two of the possible combinations are labeled (a) and (b). 
We then let $N(\mathcal{T})$ be the train track neighborhood of $\mathcal{T}$. By Roberts' construction, $N(\mathcal{T})$ fully carries a sublamination of $\mathcal{F} \cap T(\partial N)$. Without loss of generality, this sublamination $C$ satisfies the following:

(i) $C$ is a finite union of closed curves of slope $-1 / k$, where $k$ is a positive integer.

(ii) The horizontal boundary of $N(\mathcal{T})$ is contained in $C$.

Recall that $\lambda$ is oriented as $\partial S$, and is directed by $\partial / \partial x$. Orient $\mu$ so that $\dot{\mu}$ has positive $\partial / \partial y$-component. Orient $\mathcal{T}$ (and hence $C$ ) using the transverse vector field $X$. More precisely, $(\dot{C}, X)$ are to form an oriented basis for $\partial N$.

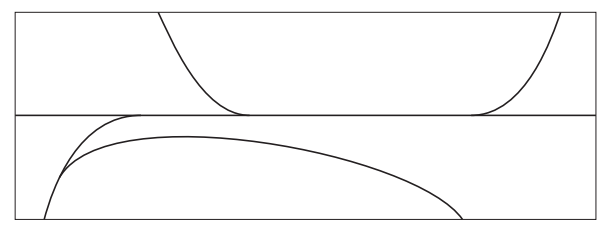

$\left(a_{1}\right)$

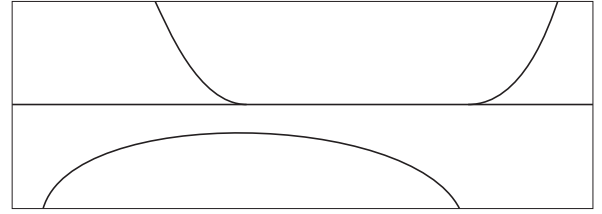

$\left(a_{2}\right)$

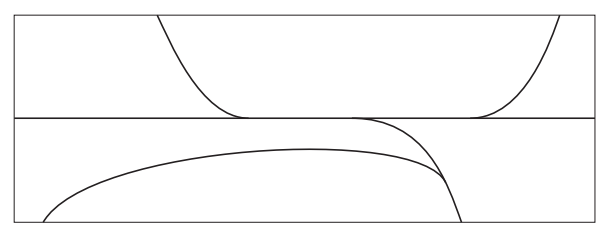

$\left(\mathrm{a}_{3}\right)$

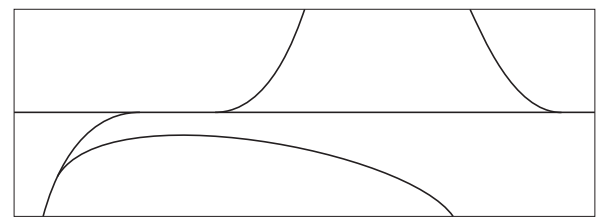

$\left(b_{1}\right)$

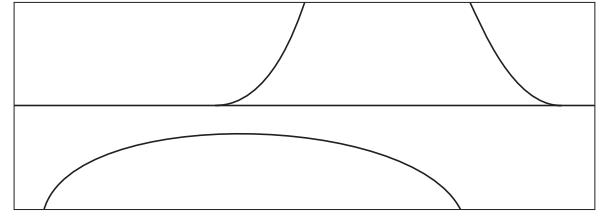

$\left(b_{2}\right)$

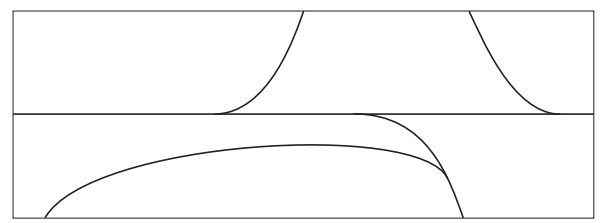

$\left(b_{3}\right)$

Figure 5: Possible splittings of $\mathcal{T}$

We now split $\mathcal{T}$ by pushing in one of the branches in $[0,1] \times[-\varepsilon, 0]$ to obtain $\mathcal{T}_{1}$, which also fully carries $C$ and satisfies (i) and (ii) above. The possibilities are given in Figure 5. We claim that $\left(a_{1}\right),\left(a_{2}\right),\left(b_{1}\right)$, or $\left(b_{2}\right)$ are not possible for $\mathcal{T}_{1}$. Indeed, in $\left(\mathrm{a}_{2}\right)$ and $\left(\mathrm{b}_{2}\right)$ the algebraic intersection number $\langle\lambda, C\rangle=0$, so $\operatorname{slope}(C)=0$, a contradiction. In cases $\left(\mathrm{a}_{1}\right)$ and $\left(\mathrm{b}_{1}\right)$, we have $\langle\lambda, C\rangle>0$ and $\langle\mu, C\rangle<0$, implying 
that slope $(C)>0$, which is also a contradiction. Therefore, $\mathcal{T}_{1}$ must be $\left(\mathrm{a}_{3}\right)$ or $\left(\mathrm{b}_{3}\right)$. Since $\mathcal{T}_{1}$ fully carries $C$, in either case there must exist a subarc $\delta:[0,1] \rightarrow \mathbb{R}^{2} / \mathbb{Z}^{2}$ of the horizontal boundary of $N\left(\mathcal{T}_{1}\right)$ such that $\delta(0)$ and $\delta(1)$ have the same $x$-coordinate, $\delta$ "winds around" in the (positive) $x$-direction once, and the $y$-coordinate of $\delta(1)$ is smaller than that of $\delta(0)$ (here we are in $[0,1] \times[-\varepsilon, \varepsilon]$ ). Let $\delta_{1}$ be the oriented integral subarc of $X=\partial / \partial y$ in $[0,1] \times[-\varepsilon, \varepsilon]$ from $\delta(1)$ to $\delta(0)$. The concatenation $\delta * \delta_{1}$ can easily be perturbed into a closed curve which is isotopic to $\lambda=\partial(S \times\{0\})$ and is positively transverse to $\mathcal{F}$. Moreover, it is easy to take the isotopy to be transverse to $X$ throughout.

Next, following Eliashberg and Thurston [2], take a $C^{0}$-small perturbation of $\mathcal{F}$, which we denote by $\xi_{\mathcal{F}}$. The characteristic foliation of $\xi_{\mathcal{F}}$ on $\partial N$ can be taken to have slope $-1 / k+\varepsilon$, where $\varepsilon$ is an arbitrarily small positive number. We can choose the perturbation so that the characteristic foliation is nonsingular Morse-Smale with two closed orbits, one attracting and one repelling, and $-1 / k+\varepsilon$ is the slope of the closed orbits. (Hence $\partial N$ is a convex surface with two dividing curves of slope $-1 / k+\varepsilon$.) We make the perturbation $\xi_{\mathcal{F}}$ sufficiently close to $\mathcal{F}$ so that $\partial\left(\phi_{1}(S)\right)$ is positively transverse to $\xi_{\mathcal{F}}$.

Since $X$ is positively transverse to $\xi_{\mathcal{F}}$ and also to $S^{\prime}:=\phi_{1}(S)$, it follows that the characteristic foliation of $\xi_{\mathcal{F}}$ on $S^{\prime}$ does not have any negative singular points. Therefore,

$$
l\left(\partial S^{\prime}\right)=-e^{+}+h^{+}+e^{-}-h^{-}=-e^{+}+h^{+}=-\chi\left(S^{\prime}\right)=2 g\left(S^{\prime}\right)-1 .
$$

where $l\left(\partial S^{\prime}\right)$ denotes the self-linking number of the transverse knot $\partial S^{\prime}$ with respect to $S^{\prime}, e^{ \pm}$and $h^{ \pm}$are the numbers of positive and negative elliptic and hyperbolic tangencies of the contact structure on $S^{\prime}$, and the genus $g(\Sigma)$ of a compact surface $\Sigma$ with boundary is the genus of the closed surface obtained by capping off all the boundary components with disks. A good reference for invariants of transverse and Legendrian knots is Etnyre [3].

Remark The fact that $X$ is positively transverse to both $S^{\prime}$ and $\xi_{\mathcal{F}}$ does not imply that the dividing set $\Gamma_{S^{\prime}}$ is empty. Although there are no negative singular points in the characteristic foliation, closed orbits of Morse-Smale type can function as sinks. Hence we can have annular regions of $S_{-}^{\prime}$, where $S^{\prime} \backslash \Gamma_{S^{\prime}}=S_{+}^{\prime} \cup S_{-}^{\prime}$.

We next explain how to pass from $S^{\prime}$ to a convex surface $S^{\prime \prime}$ with Legendrian boundary.

Lemma 4.6 There exists a convex surface $T$ isotopic to $\partial N$ inside an $I$-invariant neighborhood of $\partial N$ so that $\Gamma_{T}=\Gamma_{\partial N}$, and a convex surface $S^{\prime \prime}$ with Legendrian 
boundary (isotopic to $S^{\prime}$ ) so that $\partial S^{\prime \prime} \subset T$ and

$$
t b\left(\partial S^{\prime \prime}\right)-r\left(\partial S^{\prime \prime}\right)=l\left(\partial S^{\prime}\right)=2 g\left(S^{\prime}\right)-1 .
$$

Here $t b\left(\partial S^{\prime \prime}\right)$ and $r\left(\partial S^{\prime \prime}\right)$ are the Thurston-Bennequin invariant and rotation number of the Legendrian knot $\partial S^{\prime \prime}$ with respect to $\partial S^{\prime \prime}$.

Proof We isotop $\partial N$ inside its invariant neighborhood to obtain the convex surface $T$. More explicitly, we tilt $\partial N$ near the closed orbits of the nonsingular Morse-Smale characteristic foliation so that $T$ has Legendrian divides in place of closed orbits. Then isotop $S^{\prime}$ to $S^{\prime \prime}$ which has Legendrian boundary and such that $\partial S^{\prime \prime} \subset T$. It is not hard to see that the positive transverse push-off of $\partial S^{\prime \prime}$ is transversely isotopic to $\partial S^{\prime}$. Finally recall that if $\gamma^{+}$is a positive transverse push-off of $\gamma$, then $t b(\gamma)-r(\gamma)=l\left(\gamma^{+}\right)$ (note the sign in front of $r(\gamma)$ is negative, not positive).

Now recall that

$$
r\left(\partial S^{\prime \prime}\right)=\chi\left(S_{+}^{\prime \prime}\right)-\chi\left(S_{-}^{\prime \prime}\right)
$$

where $S_{+}^{\prime \prime}$ and $S_{-}^{\prime \prime}$ are the positive and negative regions of $S^{\prime \prime}-\Gamma_{S^{\prime \prime}}$. By comparison with Lemma 4.6, which states that:

$$
r\left(\partial S^{\prime \prime}\right)=1-2 g\left(S^{\prime \prime}\right)+t b\left(\partial S^{\prime \prime}\right),
$$

we have $\chi\left(S_{+}^{\prime \prime}\right)=1-2 g\left(S^{\prime \prime}\right)$ and $-\chi\left(S_{-}^{\prime \prime}\right)=t b\left(\partial S^{\prime \prime}\right)$. This implies the following:

Corollary 4.7 $\Gamma_{S^{\prime \prime}}$ consists of $\partial$-parallel dividing arcs and curves, together with pairs of parallel essential closed curves.

Note here that by a $\partial$-parallel dividing arc we mean a properly embedded arc that cuts off a disk whose interior intersects no other components of the dividing set. In particular, the disks cut off by $\partial$-parallel dividing arcs are disjoint. A $\partial$-parallel closed dividing curve is a closed curve parallel to the boundary.

Let us now rename $\partial N$ and $S=S \times\{1\}$ so that the following hold:

(i) $\partial N$ is convex, $\# \Gamma_{\partial N}=2$, and slope $\left(\Gamma_{\partial N}\right)=-1 / k+\varepsilon$, where $\varepsilon$ is a small positive number.

(ii) $S=S \times\{1\}$ has boundary on $\partial N$, and $\Gamma_{S}$ consists of $\partial$-parallel dividing arcs and closed curves, together with pairs of parallel essential closed curves.

(iii) The solid torus $S^{1} \times D^{2}=M-N$ is the standard neighborhood of a Legendrian curve. 
We will now normalize $\Gamma_{S}$ in a manner similar to Section 7 of [11].

Proposition 4.8 There exists a convex surface isotopic to $S$ whose dividing set only consists of $\partial$-parallel arcs.

Proof Consider the cut-open manifold $S \times[0,1]$. Here $\Gamma_{S \times\{1\}}=\Gamma_{S}$ and $\Gamma_{S \times\{0\}}=$ $\psi\left(\Gamma_{S}\right)$. Since the monodromy map $\psi$ is pseudo-Anosov, $\Gamma_{S} \neq \psi\left(\Gamma_{S}\right)$ unless $\Gamma_{S}$ is a union of $\partial$-parallel arcs and $\partial$-parallel closed curves.

We will first reduce to the case of such a union. If $\Gamma_{S} \neq \psi\left(\Gamma_{S}\right)$, then, by Proposition 7.1 of [11], there exists a closed curve $\gamma$, possibly separating, which intersects $\Gamma_{S \times\{i\}}$, $i=0,1$, efficiently and such that $\#\left(\gamma \cap \Gamma_{S \times\{1\}}\right) \neq \#\left(\gamma \cap \Gamma_{S \times\{0\}}\right)$. Now apply the Legendrian Realization Principle to make $\gamma \times\{0,1\}$ Legendrian, and apply the Flexibility Theorem to make $\gamma \times[0,1]$ convex with Legendrian boundary. By the Imbalance Principle of [9], there must exist a bypass along $\gamma \times\{0\}$, say. Let $\mathcal{B}_{\alpha}$ be the bypass and $\alpha$ the arc of attachment for the bypass.

Note that the condition that $\gamma$ intersect $\Gamma_{S \times\{i\}}$ efficiently eliminates the possibility of a trivial bypass. Hence we have the following possibilities:

(i) If $\alpha$ intersects three distinct dividing curves, then attaching $\mathcal{B}_{\alpha}$ yields a convex surface $S^{\prime}$ isotopic to $S$ with fewer dividing curves.

(ii) If $\alpha$ starts on a dividing curve $\gamma_{1}$, passes through a parallel dividing curve $\gamma_{2}$, and ends on $\gamma_{1}$, then $\gamma_{1}$ and $\gamma_{2}$ are nonseparating, and we may apply Bypass Rotation [12] so that one of the endpoints of $\alpha$ ends on a different dividing curve $\gamma_{3}$ (here $\gamma_{3}$ may be a $\partial$-parallel arc). Then apply case (i).

(iii) Suppose $\alpha$ starts on $\gamma_{1}$, passes through a parallel $\gamma_{2}$, and ends on $\gamma_{2}$ after going around a nontrivial loop. There are two possibilities: either $\gamma_{1}$ and $\gamma_{2}$ are both separating curves or they are both nonseparating curves. If $\gamma_{1}$ and $\gamma_{2}$ are both nonseparating, then we can apply Bypass Rotation and get to (i) and reduce the number of dividing curves. If $\gamma_{1}$ and $\gamma_{2}$ are both separating, but the connected component of $S \backslash \gamma_{2}$ containing the subarc of $\alpha$ from $\gamma_{2}$ to itself has other components of $\Gamma_{S}$, then we can apply Bypass Rotation, get to (i) and reduce the number of dividing curves. Finally, if $\gamma_{1}$ and $\gamma_{2}$ are separating and $\gamma_{2}$ splits off a subsurface of $S$ which does not contain other components of $\Gamma_{S}$, then attaching $\mathcal{B}_{\alpha}$ yields a pair of parallel dividing curves which are either nonseparating or are separating but split off a strictly smaller subsurface. Hence, we can reduce the complexity in one of two ways: either by reducing number of separating curves or by reducing the genus of the separated part. By repeating this procedure, we can reduce the genus of the separated part down to 1 
in finitely many steps and force the appearance of a nonseparating pair. Then apply case (i) or (ii).

We can repeat this procedure until we eliminate all pairs of curves that are not parallel to the boundary.

To eliminate the closed curves parallel to $\partial S$, we cut $N$ open along $S$ to obtain $S \times[0,1]$. The dividing set on $\partial(S \times[0,1])$, after rounding, will consist of $4 n+1$ closed curves which are parallel to $\partial S$. There are $2 n$ each on $S \times\{0\}$ and $S \times\{1\}$, and one which is created from the $\partial$-parallel arcs by edge-rounding. Let us number the dividing curves consecutively (as they appear on $\partial(S \times[0,1]))$ as $\gamma_{1}, \ldots, \gamma_{4 n+1}$. Now let $\delta$ be a properly embedded, non-boundary-parallel arc from $\partial S$ to itself. Then cut $S \times[0,1]$ along the disk $D=\delta \times[0,1]$, which we take to be convex with Legendrian boundary. Furthermore, we take $\partial D$ to be efficient with respect to $\Gamma_{\partial(S \times[0,1])}$. Now consider the $\partial$-parallel arcs of $\Gamma_{D}$. The only time a $\partial$-parallel arc does not have a corresponding bypass which reduces $\# \Gamma_{S \times\{0\}}$ or $\# \Gamma_{S \times\{1\}}$ or puts us in case (iii) above is if it straddled the middle curve $\gamma_{2 n+1}$. In this case, there are only two $\partial$-parallel arcs on $D$ and all the other dividing $\operatorname{arcs}$ on $D$ are "parallel" to these $\partial$-parallel arcs that straddle $\gamma_{2 n+1}$.

However, we claim that this particular form of $\Gamma_{D}$ implies that there is a closed Legendrian curve $\delta$ which is isotopic to a meridian curve on $\partial N$ and has zero relative Thurston-Bennequin invariant with respect to the tangent framing of $\partial N$. In fact, any properly embedded Legendrian $\operatorname{arc} \delta_{i}$ on $D$ which is parallel to and disjoint from arcs of $\Gamma_{D}$, and has endpoints on $\gamma_{i}$ and $\gamma_{4 n+2-i}$, glues to give such a closed Legendrian curve $\delta$, after possibly sliding an endpoint along $\gamma_{i}$. Now, $\delta$ bounds an overtwisted disk in $M$, obtained from $N$ by Dehn filling along the meridian slope. This contradicts the fact that the contact structure $\xi_{\mathcal{F}}$ on $M$ is a tight contact structure. (Recall that $\xi_{\mathcal{F}}$ is a perturbation of a taut foliation $\mathcal{F}$.) This proves that $\Gamma_{S}$, after successive bypass attachments, can be made to consist only of $\partial$-parallel arcs.

Finally, to prove that $\xi_{\mathcal{F}}$ is the same as the contact structure defined by $(S, h)$, we cut $N$ open along $S$ with only $\partial$-parallel dividing arcs, as furnished by Proposition 4.8 , and consider the contact structure on the cut-open manifold induced by $\xi_{\mathcal{F}}$. The dividing set for $\xi_{\mathcal{F}}$ on $\partial(S \times[0,1])$ consists of one closed curve parallel to $\partial S$. We can now take a system of $\operatorname{arcs} \alpha_{i}, i=1, \ldots, 2 g(S)$, on $S$ and cut along the disks $\alpha_{i} \times[0,1]$ to decompose $S \times[0,1]$ into a disk times $[0,1]$. Note that each decomposition is along a convex disk $D_{i}$ isotopic to $\alpha_{i} \times[0,1]$ with Legendrian boundary, so that $\partial D_{i}$ intersects $\Gamma_{\partial(S \times[0,1])}$ in exactly two points. Hence the dividing set $\Gamma_{D_{i}}$ is determined, ie, consists of a single dividing arc. There is a unique (up to isotopy) contact structure with such a decomposition, called a product disk decomposition. The decomposition of 
$M$ into such an $N$ and a standard neighborhood $S^{1} \times D^{2}$ of a Legendrian curve is clearly identical to the contact structure adapted to the open book $(S, h)$.

Acknowledgements We thank Francis Bonahon and Bill Thurston for helpful discussions. Discussions with Étienne Ghys also helped clarify aspects of Section 3. We are also very grateful to the referee for the extensive list of comments.

KH supported by an Alfred P Sloan Fellowship and an NSF CAREER Award (DMS0237386); GM supported by NSF grant DMS-0410066; WHK supported by NSF grant DMS-0406158.

\section{References}

[1] J Barge, É Ghys, Cocycles d'Euler et de Maslov, Math. Ann. 294 (1992) 235-265 MR1183404

[2] Y M Eliashberg, W P Thurston, Confoliations, University Lecture Series 13, Amer. Math. Soc. (1998) MR1483314

[3] J B Etnyre, Legendrian and transversal knots, from: "Handbook of knot theory", Elsevier B. V., Amsterdam (2005) 105-185 MR2179261

[4] J-M Gambaudo, É Ghys, Commutators and diffeomorphisms of surfaces, Ergodic Theory Dynam. Systems 24 (2004) 1591-1617 MR2104597

[5] J-M Gambaudo, É Ghys, Braids and signatures, Bull. Soc. Math. France 133 (2005) 541-579 MR2233695

[6] É Ghys, Groups acting on the circle, Enseign. Math. (2) 47 (2001) 329-407 MR1876932

[7] E Giroux, Géométrie de contact: de la dimension trois vers les dimensions supérieures, from: "Proc. of the ICM, Vol. II (Beijing, 2002)", Higher Ed. Press, Beijing (2002) 405-414 MR1957051

[8] A Hatcher, Some examples of essential laminations in 3-manifolds, Ann. Inst. Fourier (Grenoble) 42 (1992) 313-325 MR1162564

[9] K Honda, On the classification of tight contact structures. I, Geom. Topol. 4 (2000) 309-368 MR1786111

[10] K Honda, W H Kazez, G Matić, On the contact class in Heegaard Floer homology arXiv:math.GT/0609734

[11] K Honda, W H Kazez, G Matić, Tight contact structures on fibered hyperbolic 3manifolds, J. Differential Geom. 64 (2003) 305-358 MR2029907

[12] K Honda, W H Kazez, G Matić, Right-veering diffeomorphisms of compact surfaces with boundary, Invent. Math. 169 (2007) 427-449 MR2318562 
[13] S Y Orevkov, Markov moves for quasipositive braids, C. R. Acad. Sci. Paris Sér. I Math. 331 (2000) 557-562 MR1794098

[14] J Robbin, D Salamon, The Maslov index for paths, Topology 32 (1993) 827-844 MR1241874

[15] R Roberts, Taut foliations in punctured surface bundles. I, Proc. London Math. Soc. (3) 82 (2001) 747-768 MR1816696

[16] R Roberts, Taut foliations in punctured surface bundles. II, Proc. London Math. Soc. (3) 83 (2001) 443-471 MR1839461

[17] W P Thurston, HE Winkelnkemper, On the existence of contact forms, Proc. Amer. Math. Soc. 52 (1975) 345-347 MR0375366

KH: Department of Mathematics, University of Southern California

Los Angeles, CA 90089, USA

WHK, GM: Department of Mathematics, University of Georgia

Athens, GA 30602, USA

khonda@math.usc.edu, will@math.uga.edu, gordana@math.uga.edu

http://rcf.usc.edu/ khonda, http://www.math.uga.edu/ will,

http://www. math.uga.edu/ gordana

Proposed: Yasha Eliashberg

Received: 6 December 2006

Seconded: Peter Ozsváth, Tom Mrowka

Revised: 22 April 2008 